\title{
Thioxopyrimidine in Heterocyclic Synthesis I: Synthesis of Some Novel 6-(Heteroatom-substituted)-(thio)pyrimidine Derivatives
}

\author{
Yuh-Wen Ho and Maw Cherng Suen \\ Department of Creative Fashion Design, Taoyuan Innovation Institute of Technology, Jhongli 32091, Taiwan \\ Correspondence should be addressed to Yuh-Wen Ho; wen@tiit.edu.tw
}

Received 27 June 2012; Revised 30 August 2012; Accepted 16 October 2012

Academic Editor: Filomena Conforti

Copyright (c) 2013 Y.-W. Ho and M. C. Suen. This is an open access article distributed under the Creative Commons Attribution License, which permits unrestricted use, distribution, and reproduction in any medium, provided the original work is properly cited.

A series of novel $\mathrm{N}$-cycloalkanes, morpholine, piperazines, pyrazole, pyrimidine, benzimidazolo[1,2-a]pyrimidine, 1,2,3,4tetrazolo[1,5-a]pyrimidine, azopyrazolo[1,5- a]pyrimidine, pyrimido[4, 5':3,4]pyrazolo[1,5-a]pyrimidines and pyridine derivatives incorporating a 5-cyano-4-methyl-2-phenyl-(thio)pyrimidine moiety were obtained by the intramolecular cyclization of 6methylthio-pyrimidine, 6-(benzoylmethyl)thio- pyrimidine and 2-[(5-cyano-4-methyl-2-phenylpyrimidin-6-yl)thio]-3-dimethylamino-1-phenyl-prop-2-en-1-one with appropriate amines and enaminone compounds, respectively. The structure of all new synthesized compounds was established from their spectral data, elemental analysis and the X-ray crystal analysis.

\section{Introduction}

Pyrimidine derivatives attracted organic chemists very much due to their biological and chemotherapeutic importance. Pyrimidine derivatives and related fused heterocycles are important classes of heterocyclic compounds that exhibit a broad spectrum of biological activities such as anticancer [15], antiviral [6], antibacterial $[7,8]$, antioxidant $[9,10]$, anxiolytic [11], and antidepressant activities [12]. Furthermore, they possess anti-inflammatory [13-19] and analgesic activities that are well documented in the literature [20-22]. The incorporation of two moieties increases biological activity of both and thus it was of value to synthesize some new heterocyclic derivatives having two moieties in the same molecules. The course of our researches was devoted to the development of new classes of pyrimidines substituted at position- 6 with different fused heterocycles moiety in the hope that they may be biologically active. In preceding papers [23-25] we have described the synthesis of a series of novel 5-(1-pyrrolyl)4-methyl-2-phenylthieno[2,3-d]pyrimidine derivatives containing chalcones, pyridines, pyridin-2(1H)-ones, $2 H$ pyran-2-one, pyrazoles, pyrimidines imidazolpyrimidines, pyrazolopyrimidines, and 1,3,4-oxadiazoles moiety. In continuation of our studies, we report herein the use of thioxopyrimidine $\mathbf{1}$ for the synthesis of various N-cycloalkanes, morpholine, piperazines, pyridines, pyrazole, pyrimidine, benzimidazolo[1,5-a]pyrimidine, 1,2,3,4-tetrazolo $[1,5-a]$ pyrimidine, azopyrazolo[ $1,5-a]$ pyrimidine, and pyrimido $\left[4^{\prime}, 5^{\prime}: 3,4\right]$ pyrazolo $[1,5-a]$ pyrimidine incorporating a (thio)pyrimidine moiety. The structure of the new compounds was verified by spectroscopic methods and the X-ray crystal structure of compound $\mathbf{1 3}$ is also discussed.

\section{Experimental}

All melting points are uncorrected and in ${ }^{\circ} \mathrm{C}$. IR spectra were recorded on a JASCO FTIR-3 spectrometer $(\mathrm{KBr}) ;{ }^{1} \mathrm{H}$ NMR spectra were obtained on a Bruker AM-300 WB FINLR spectrometer, and chemical shifts are expressed in $\delta$ ppm using TMS as an internal standard. Electron impact mass spectra were obtained at $70 \mathrm{eV}$ using a Finingan Mat TSQ-46C spectrometer. Microanalyses for C, $\mathrm{H}$, and $\mathrm{N}$ were performed on a Perkin-Elmer 240 elemental Analyzer. Enaminone derivatives 14a-f, 5-amino-4-phenylazo3-methyl-1H-pyrazole 26, and 3-amino-4-methyl-6-phenylpyrazolo-[3,4- $d$ ] pyrimidine 28 were prepared following the methods in the literature $[24,26,27]$. 


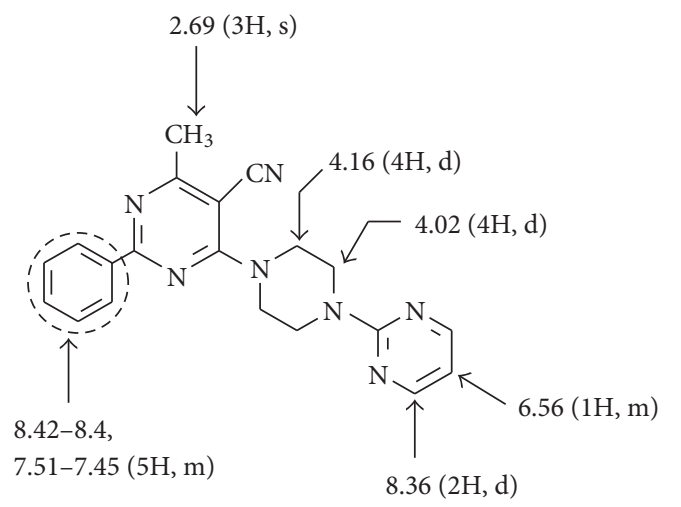

FIGURE 1: Structural assignment of typical protons in $\mathbf{5} \mathbf{f}$ by ${ }^{1} \mathrm{H}$ NMR.

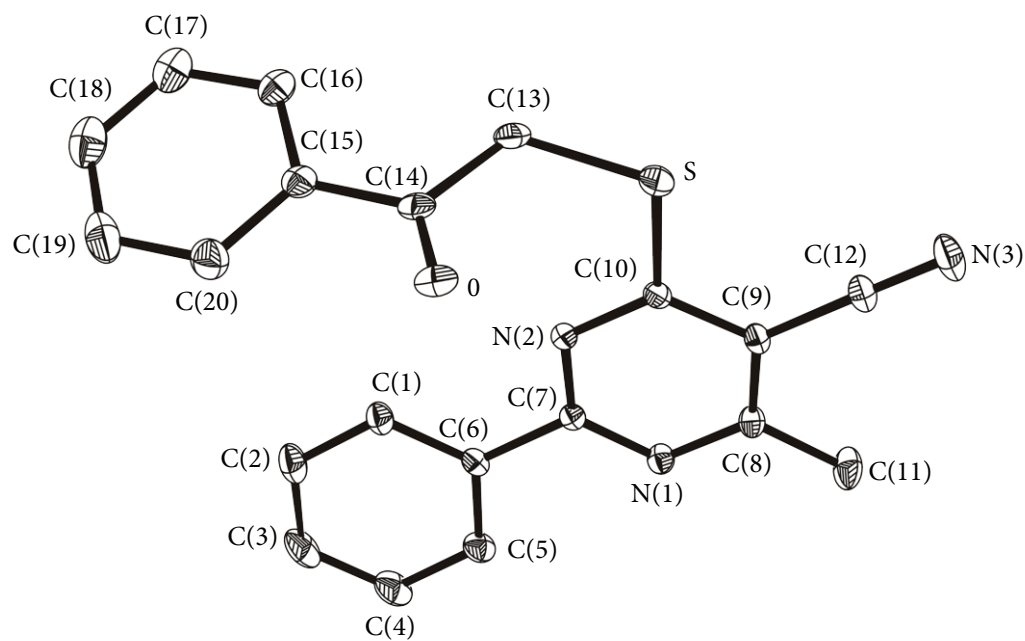

FIgURE 2: Perspective view of compound $\mathbf{1 3}$ with atomic numbering.

2.1. 5-Cyano-1,6-dihydro-4-methyl-2-phenyl-6-thioxopyrimidine (1). To a suspension of ammonium thiocyanate $(7.60 \mathrm{~g}, 0.1 \mathrm{~mol})$ in dry dioxane $(100 \mathrm{~mL})$, benzoyl chloride ( $14 \mathrm{~g}, 0.1 \mathrm{~mol}$ ) was added. The reaction mixture was refluxed for $5 \mathrm{~min}$., then treated with 3 -aminocrotononitrile $(8.20 \mathrm{~g}$, $0.1 \mathrm{~mol})$. The reaction mixture was refluxed for $2 \mathrm{~h}$ and poured into ice water. The solid product was collected by filtration, washed with water, and recrystallized from ethanol to give $17 \mathrm{~g}$ of yellow needle crystals (74\% yield), mp 212 ${ }^{\circ}$; IR: $v 2225(\mathrm{CN}), 1200(\mathrm{C}=\mathrm{S}) \mathrm{cm}^{-1}$; ${ }^{1} \mathrm{H}-\mathrm{NMR}$ $\left(\mathrm{DMSO}-d_{6}\right): \delta 2.50\left(3 \mathrm{H}, \mathrm{s}, \mathrm{CH}_{3}\right), 2.90(1 \mathrm{H}, \mathrm{s}, \mathrm{NH}), 7.66-7.51$, 8.11-8.08 (5H, m, phenyl-H); $m / z: 227\left(\mathrm{M}^{+}\right)$. Anal. Calcd. for $\mathrm{C}_{12} \mathrm{H}_{9} \mathrm{~N}_{3} \mathrm{~S}$ : C, 63.43; H, 3.96; N, 18.50. Found: C, 63.40; $\mathrm{H}, 4.00 ; \mathrm{N}, 18.60 \%$.

2.2.5-Cyano-4-methyl-2-phenyl-6-methylthio-pyrimidine(2). A mixture of compound $1(0.23 \mathrm{~g}, 1 \mathrm{mmol})$ in methanol $(10 \mathrm{~mL})$ and sodium methoxide $(0.08 \mathrm{~g}, 1.5 \mathrm{mmol})$ and methyl iodide $(0.17 \mathrm{~g}, 1.2 \mathrm{mmol})$ were added. After stirring at room temperature for $4 \mathrm{~h}$, the resulting solid product was collected by filtration, washed with water, and recrystallized from THF to give $0.21 \mathrm{~g}$ of pale yellow crystals ( $87 \%$ yield), mp $144^{\circ} \mathrm{C}$; IR: $v 2207(\mathrm{C} \equiv \mathrm{N}) \mathrm{cm}^{-1}{ }^{1} \mathrm{H}-\mathrm{NMR}\left(\mathrm{CDCl}_{3}\right): \delta 2.70$ $\left(3 \mathrm{H}, \mathrm{s}, \mathrm{CH}_{3}\right), 2.75\left(3 \mathrm{H}, \mathrm{s}, \mathrm{SCH}_{3}\right), 8.49-8.47,7.55-7.49(5 \mathrm{H}$, $\mathrm{m}$, phenyl-H); MS (m/z, \%): 241( $\left.\mathrm{M}^{+}, 100\right), 227(4), 195(4)$, 153(14), 138(9), 111(3), 104(17), 244(2), 77(10), 51(6). Anal. Calcd. for $\mathrm{C}_{13} \mathrm{H}_{11} \mathrm{~N}_{3} \mathrm{~S}$ : C, 64.73; H, 4.56; N, 17.42. Found: C, $64.88 \mathrm{H}, 4.54 ; \mathrm{N}, 17.55 \%$.

2.3. 5-Cyano-4-formyl-2-phenyl-6-methylthio-pyrimidine (3). A mixture of 5-cyano-4-methyl-2-phenyl-6-methylthio-pyrimidine $2(0.24 \mathrm{~g}, 1 \mathrm{mmol})$ and selenium oxide $(0.11 \mathrm{~g}$, $1 \mathrm{mmol})$ in dioxane $(10 \mathrm{~mL})$ was refluxed for $1 \mathrm{~h}$. After cooling, the mixture was filtered. The filtrate was evaporated under reduced pressure, and the resulting residue was purified by column chromatography (light petroleum ether/ethyl acetate $7: 3 \mathrm{v} / \mathrm{v}$ as eluent) to give $0.2 \mathrm{~g}$ of reddish brown needles (78\% yield), mp $164^{\circ} \mathrm{C}$; IR: $v 2218(\mathrm{C} \equiv \mathrm{N}), 1689$ $(\mathrm{C}=\mathrm{O}) \mathrm{cm}^{-1} ;{ }^{1} \mathrm{H}-\mathrm{NMR}\left(\mathrm{DMSO}-d_{6}\right): \delta 2.74\left(3 \mathrm{H}, \mathrm{s}, \mathrm{SCH}_{3}\right)$, 8.40, 7.61-7.52 (5H, m, phenyl-H), 9.85 (1H, s, CHO); MS ( $m / z, \%): 255\left(\mathrm{M}^{+}, 18\right), 241(100), 277(61), 200(22), 138(10)$, 


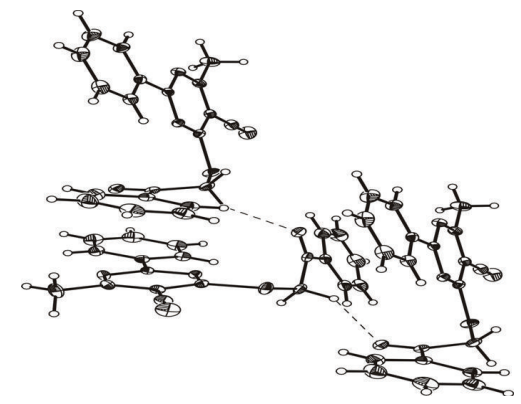

(a)

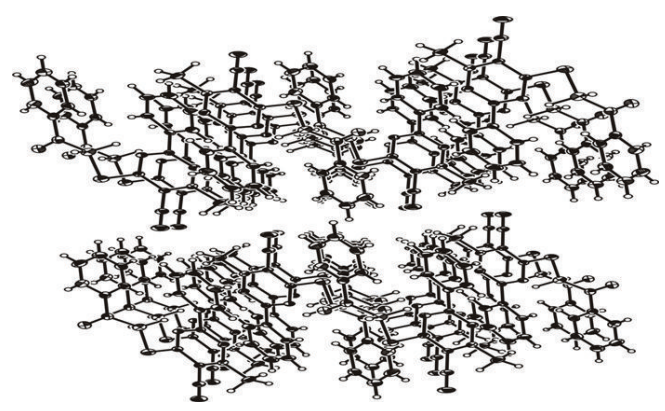

(b)

Figure 3: (a) Packing diagram for 13 showing the $\mathrm{C}-\mathrm{H}-\mathrm{O}$ hydrogen bonds $\left(\mathrm{C}-\mathrm{H}-\mathrm{O}=2.261 \AA\right.$, $\left.\angle \mathrm{C}-\mathrm{H}-\mathrm{O}=145.5^{\circ}\right)$. (b) Packing diagram for $\mathbf{1 3}$ showing the interactions between the layers.

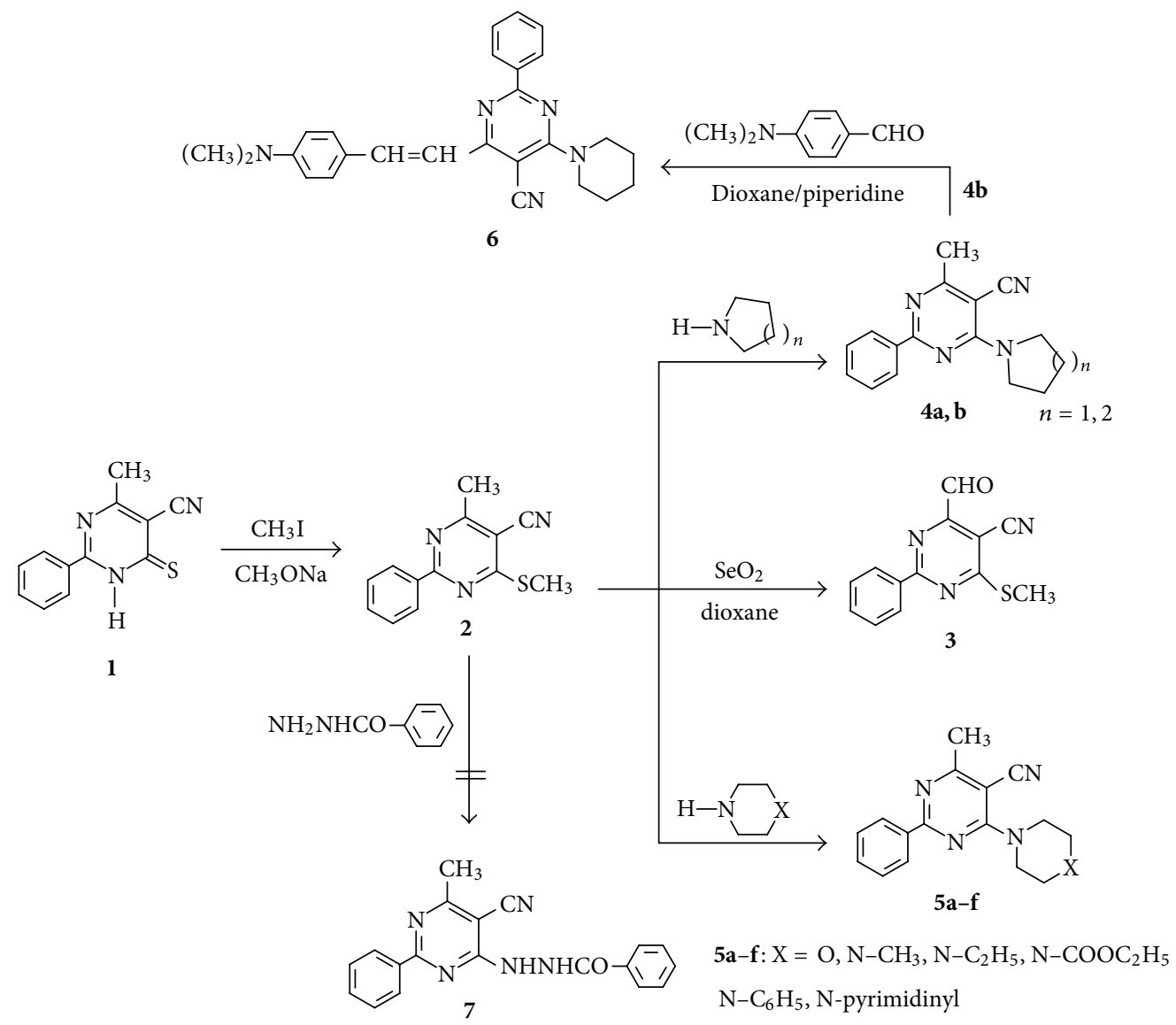

SCHEME 1

104(12), 97(5). Anal. Calcd. for $\mathrm{C}_{13} \mathrm{H}_{9} \mathrm{~N}_{3}$ OS: C, 61.17; $\mathrm{H}$, $3.52 ; \mathrm{N}, 16.47$. Found: C, 61.13; H, 3.72; N, 16.45\%.

2.4. 6-Substituted-5-cyano-4-methyl-2-phenyl-pyrimidine Derivatives (4a,b and 5a-f) General Procedure. A mixture of compound $2(0.24 \mathrm{~g}, 1 \mathrm{mmol})$ and excess secondary amines (pyrrolidine, piperidine, morpholine, N-methylpiperazine,
N-ethylpiperazine, ethyl 1-piperazinecarboxylate, 1-phenylpiperazine, and 1-(2-pyrimidyl)piperazine) (5 mmol) was refluxed for $10 \mathrm{~h}$ and poured into ice-water, and the precipitated product was collected by filtration, washed with water, and the crude product recrystallized from chloroform/THF. The physical constants and spectral data of compounds $\mathbf{4 a}, \mathbf{b}$ and $\mathbf{5 a}-\mathbf{f}$ are recorded in Tables 1 and 2 . 

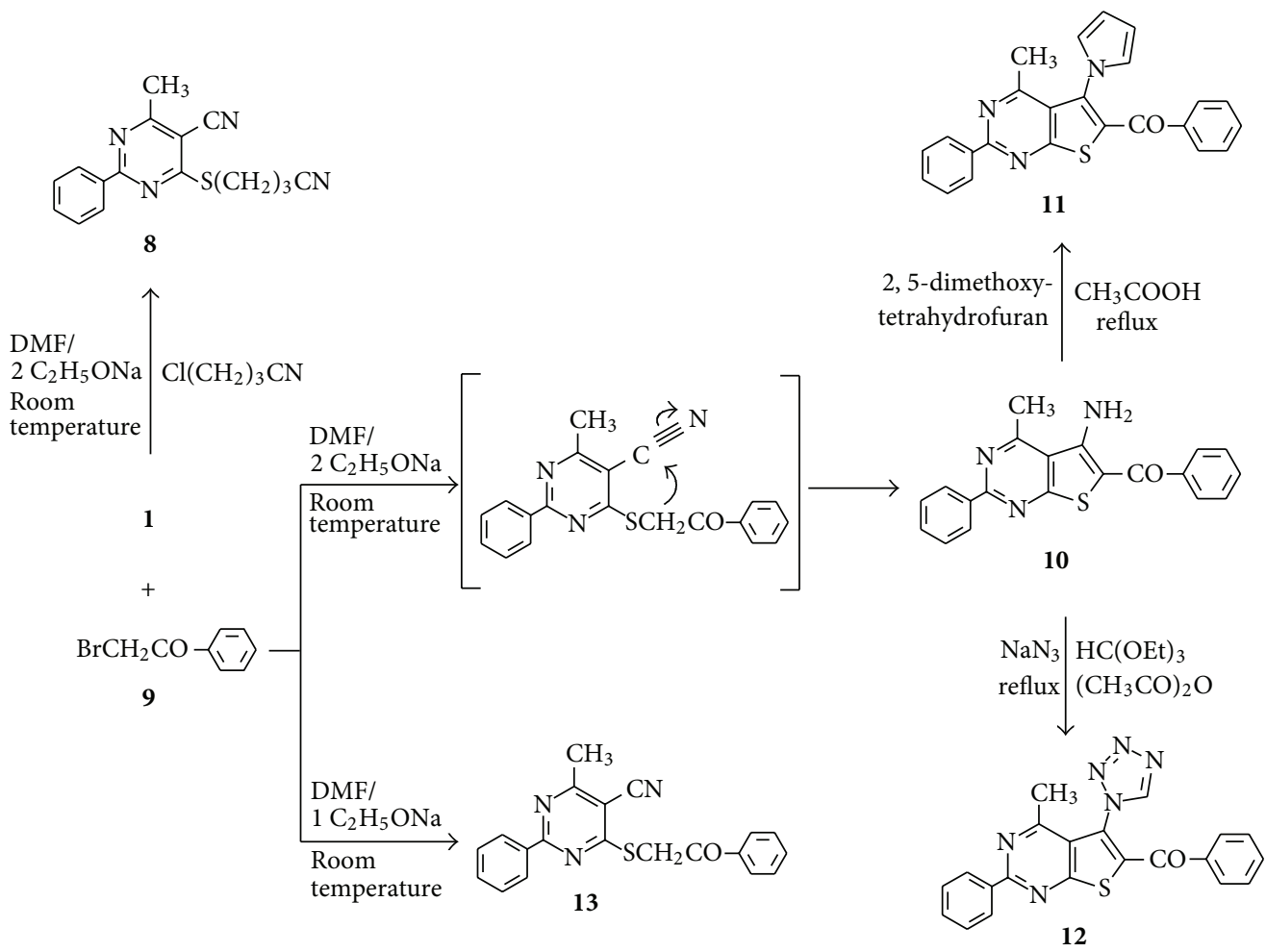

SCHEMe 2

TABLE 1: Physical and analytical data of 6-substituted-pyrimidine derivatives (4a, b and $\mathbf{5 a}-\mathbf{f})$.

\begin{tabular}{|c|c|c|c|c|c|c|c|}
\hline \multirow{2}{*}{ Compd. } & \multirow{2}{*}{$\mathrm{R}$} & \multirow{2}{*}{ M.P. $\left({ }^{\circ} \mathrm{C}\right)^{\mathrm{a}}$} & \multirow{2}{*}{ Yield (\%) } & \multirow{2}{*}{ Molecular formula } & \multicolumn{3}{|c|}{ Elemental Analysis (\%) Calcd/Found. } \\
\hline & & & & & $\mathrm{C}$ & $\mathrm{H}$ & $\mathrm{N}$ \\
\hline \multirow[t]{2}{*}{$4 a$} & $\mathrm{~N}$ & \multirow{2}{*}{154} & \multirow{2}{*}{89} & \multirow{2}{*}{$\mathrm{C}_{16} \mathrm{H}_{16} \mathrm{~N}_{4}$} & 72.72 & 6.06 & 21.21 \\
\hline & & & & & 72.79 & 6.01 & 21.24 \\
\hline \multirow[t]{2}{*}{$4 b$} & $=2$ & \multirow[t]{2}{*}{111} & \multirow[t]{2}{*}{88} & \multirow{2}{*}{$\mathrm{C}_{17} \mathrm{H}_{18} \mathrm{~N}_{4}$} & 73.38 & 6.47 & 20.14 \\
\hline & & & & & 73.41 & 6.51 & 20.11 \\
\hline \multirow[t]{2}{*}{$5 \mathbf{a}$} & & \multirow{2}{*}{178} & \multirow{2}{*}{79} & \multirow{2}{*}{$\mathrm{C}_{16} \mathrm{H}_{16} \mathrm{~N}_{4} \mathrm{O}$} & 68.57 & 5.71 & 20.00 \\
\hline & & & & & 68.59 & 5.72 & 19.98 \\
\hline \multirow{2}{*}{$5 \mathbf{b}$} & $-\mathrm{CH}_{3}$ & \multirow{2}{*}{130} & \multirow{2}{*}{28} & \multirow{2}{*}{$\mathrm{C}_{17} \mathrm{H}_{19} \mathrm{~N}_{5}$} & 69.62 & 6.48 & 23.89 \\
\hline & & & & & 69.58 & 6.58 & 23.88 \\
\hline \multirow{2}{*}{$5 c$} & ${ }_{2} \mathrm{H}_{5}$ & \multirow{2}{*}{118} & \multirow{2}{*}{87} & \multirow{2}{*}{$\mathrm{C}_{18} \mathrm{H}_{21} \mathrm{~N}_{5}$} & 70.35 & 6.84 & 22.80 \\
\hline & & & & & 70.39 & 5.78 & 22.75 \\
\hline \multirow[t]{2}{*}{$5 d$} & $\mathrm{OOC}_{2} \mathrm{H}_{5}$ & \multirow{2}{*}{174} & \multirow[t]{2}{*}{71} & \multirow{2}{*}{$\mathrm{C}_{19} \mathrm{H}_{21} \mathrm{~N}_{5} \mathrm{O}_{2}$} & 64.95 & 5.98 & 19.94 \\
\hline & & & & & 65.04 & 6.02 & 20.01 \\
\hline \multirow[t]{2}{*}{$5 e$} & $\mathrm{~N}$ & \multirow[t]{2}{*}{164} & \multirow[t]{2}{*}{67} & \multirow{2}{*}{$\mathrm{C}_{22} \mathrm{H}_{21} \mathrm{~N}_{5}$} & 74.36 & 5.91 & 19.71 \\
\hline & & & & & 74.28 & 6.09 & 19.88 \\
\hline \multirow[t]{2}{*}{$5 f$} & $\mathrm{~N}$ & \multirow{2}{*}{185} & 64 & $\mathrm{C}_{2} \mathrm{H}_{10} \mathrm{~N}_{-}$ & 67.22 & 5.32 & 27.45 \\
\hline & & & & & 67.39 & 5.14 & 27.61 \\
\hline
\end{tabular}

${ }^{\text {a }}$ Recrystallization from $\mathrm{CHCl}_{3} / \mathrm{THF}$. 


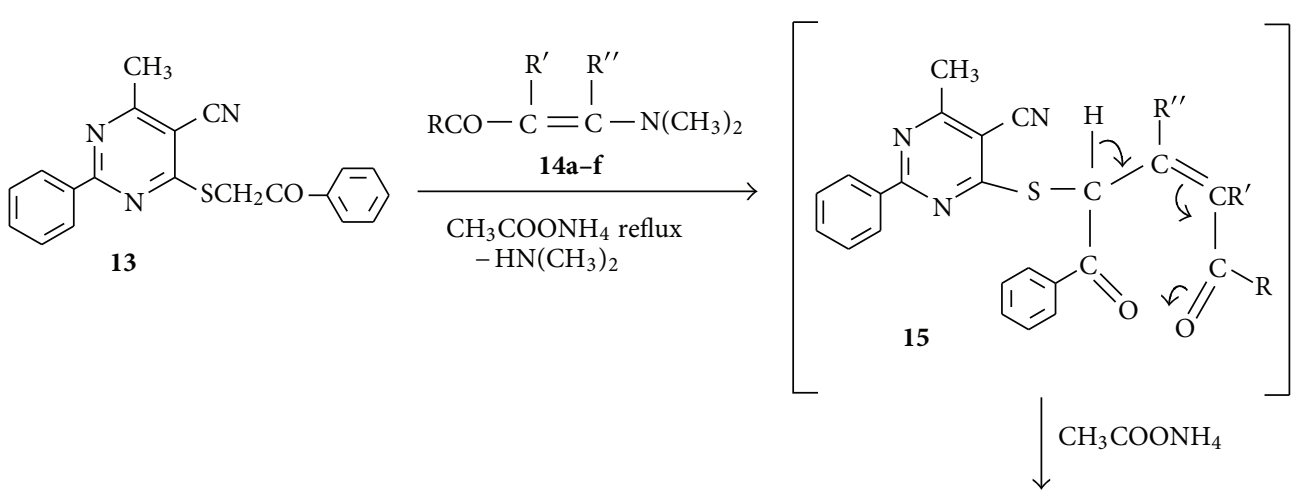

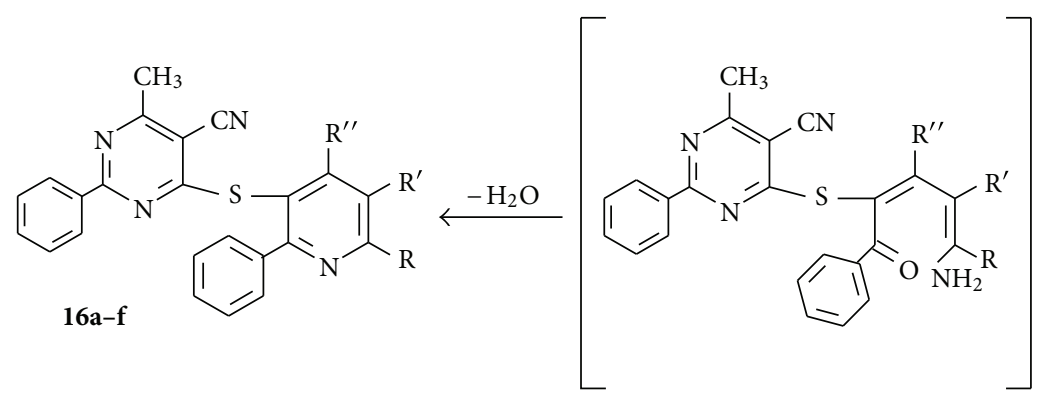

\begin{tabular}{|c|c|c|c|c|c|c|}
\hline 14,16 & $\mathbf{a}$ & b & c & d & e & $\mathrm{f}$ \\
\hline $\mathrm{R}$ & & & & & 1 & $\mathrm{CH}$ \\
\hline $\mathrm{R}^{\prime}$ & $\mathrm{H}$ & $\mathrm{H}$ & $\mathrm{H}$ & $\mathrm{CN}$ & $\mathrm{H}$ & $C \Omega$ \\
\hline $\mathrm{R}^{\prime \prime}$ & $\mathrm{H}$ & $\mathrm{H}$ & $\mathrm{H}$ & $\mathrm{H}$ & $\mathrm{CH}_{3}$ & $\mathrm{H}$ \\
\hline
\end{tabular}

2.5. 4-[(4-N,N-Dimethylamino)-2-phenylvinyl)]-6-piperidinyl-5-cyano-2-phenyl-pyrimidine (6). A mixture of 6-piperidinyl-4-methyl-pyrimidine $4 \mathbf{b}(0.28 \mathrm{~g}, 1 \mathrm{mmol})$ and N,Ndimethylaminobenzaldehyde $(0.15 \mathrm{~g}, 1 \mathrm{mmol})$ in dioxane $(10 \mathrm{~mL})$ in the presence of catalytic amount of piperidine was refluxed for $8 \mathrm{~h}$. After cooling, the resulting solid product was collected by filtration and washed with water, and the crude product recrystallized from ethanol/glacial acetic acid to give $0.15 \mathrm{~g}$ of pale yellow needles (37\% yield), mp $103^{\circ} \mathrm{C}$; IR: $v 2212(\mathrm{C} \equiv \mathrm{N}) \mathrm{cm}^{-1} ;{ }^{1} \mathrm{H}-\mathrm{NMR}\left(\mathrm{CDCl}_{3}\right): \delta$ 3.07-2.96, 1.75-1.62 (10H, m, piperidinyl-H), $3.09\left(6 \mathrm{H}, \mathrm{s}, \mathrm{CH}_{3}\right), 6.70$ $(1 \mathrm{H}, \mathrm{d}, J=2.0 \mathrm{~Hz},-\mathrm{CH}=), 7.74(1 \mathrm{H}, \mathrm{d}, J=2.0 \mathrm{~Hz},=\mathrm{CH}-)$, 8.57-8.55, 7.52-7.44 (9H, m, phenyl-H); MS ( $m / z, \%)$ : 409( $\left.\mathrm{M}^{+}, 100\right), 381(9), 366(3), 326(5), 289(7), 222(4), 205(6)$, 195(8), 190(5), 104(7), 85(5). Anal. Calcd. for $\mathrm{C}_{26} \mathrm{H}_{27} \mathrm{~N}_{5}$ : C, 76.28; H, 6.60; N, 17.11. Found: C, 76.01; H, 6.42; N, 17.31\%.
2.6. 6-Cyanopropylthio-5-cyano-4-methyl-2-phenyl-pyrimidin (8). A mixture of 5-cyano-1,6-dihydro-4-methyl-2phenyl-6-thioxopyrimidine $\mathbf{1}(2.27 \mathrm{~g}, 0.01 \mathrm{~mol})$, sodium ethoxide $(1.36 \mathrm{~g}, 0.02 \mathrm{~mol})$, and 4-chlorobutyronitrile $(1.03 \mathrm{~g}, 0.01 \mathrm{~mol})$ in DMF $(50 \mathrm{~mL})$. The reaction mixture was stirred at room temperature for $4 \mathrm{~h}$ and then diluted with cold water $(50 \mathrm{~mL})$ was collected by filtration, washed with water, and recrystallized from DMF/ethanol to give $2.88 \mathrm{~g}$ of yellow needles (98\% yield), mp $144^{\circ} \mathrm{C}$; IR: $v 2220(\mathrm{C} \equiv \mathrm{N})$ $\mathrm{cm}^{-1} ;{ }^{1} \mathrm{H}-\mathrm{NMR}\left(\mathrm{CDCl}_{3}\right): \delta 2.21\left(2 \mathrm{H}, \mathrm{t}, J=2.54 \mathrm{~Hz}, \mathrm{CH}_{2}\right)$, 2.58-2.56 $\left(2 \mathrm{H}, \mathrm{m}, \mathrm{CH}_{2}\right), 2.71\left(3 \mathrm{H}, \mathrm{s}, \mathrm{CH}_{3}\right), 3.54(2 \mathrm{H}, \mathrm{t}, \mathrm{J}=$ $\left.2.27 \mathrm{~Hz}, \mathrm{CH}_{2}\right), 8.47-8.46,7.55-7.49$ (5H, m, phenyl-H); MS $(\mathrm{m} / z, \%):$ 294( $\left.\mathrm{M}^{+}, 22\right), 254(60), 241(100), 227(28), 194(9)$, 153(31), 104(15), 77(16). Anal. Calcd. for $\mathrm{C}_{16} \mathrm{H}_{14} \mathrm{~N}_{4} S$ : C, 65.30; H, 4.76; N, 19.04. Found: C, 65.23; H, 4.70; N, $19.13 \%$. 


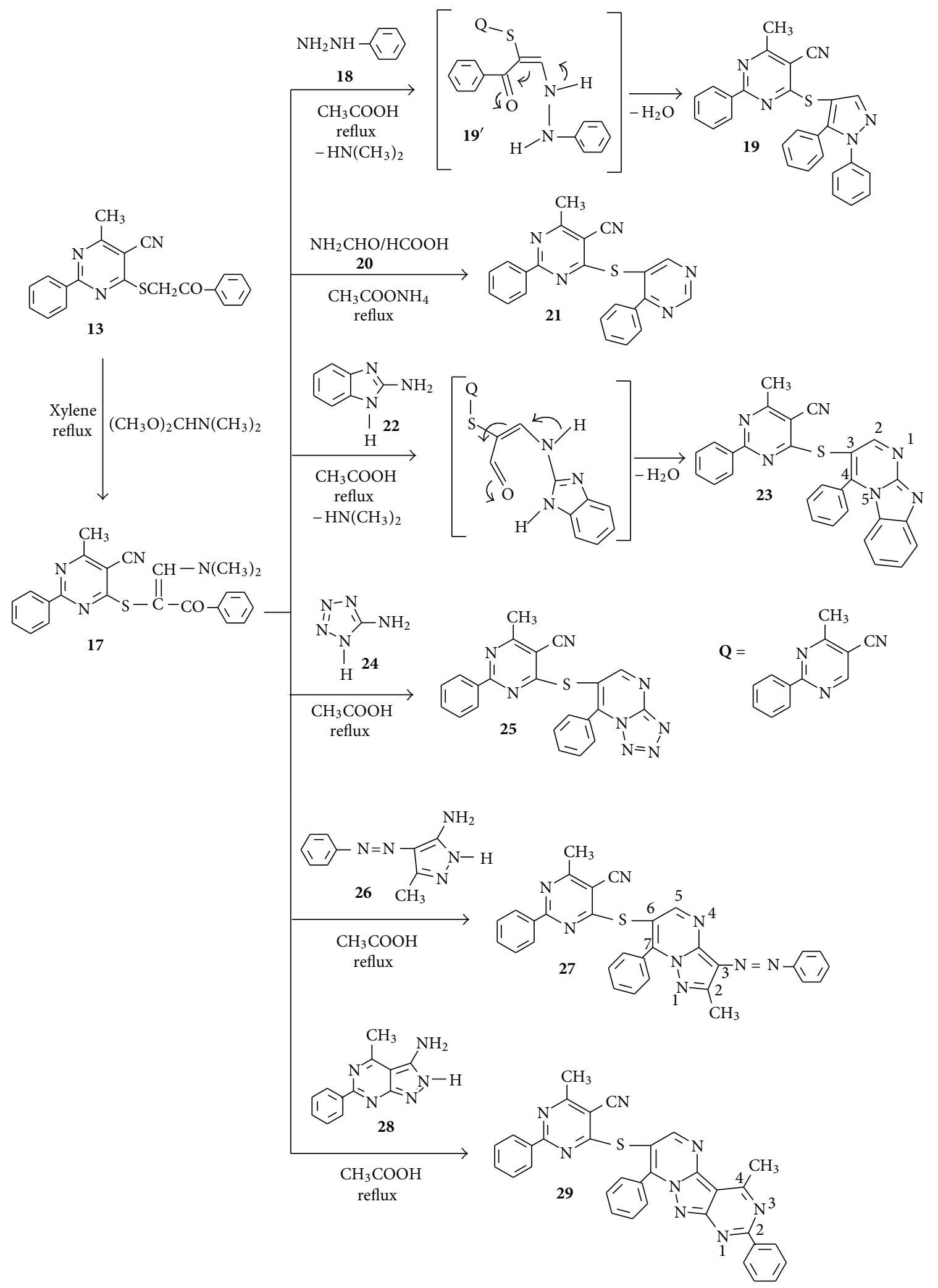

SCHEMe 4 
TABLE 2: Spectral data of 6-substituted-pyrimidine derivatives (4a, $\mathbf{b}$ and $\mathbf{5 a - f})$.

\begin{tabular}{|c|c|c|c|}
\hline Compd. & $\mathrm{MS}\left(\mathrm{m} / \mathrm{e} \mathrm{M}^{+}\right)$ & $\begin{array}{l}\mathrm{IR}(\mathrm{KBr}) \\
v\left(\mathrm{~cm}^{-1}\right)\end{array}$ & $\begin{array}{l}{ }^{1} \mathrm{H}_{-\mathrm{NMR}^{\mathrm{a}}} \\
\left(\mathrm{CDCl}_{3}\right) \\
\delta(\mathrm{ppm}) \\
\end{array}$ \\
\hline $4 a$ & 264(98), 235(100), 209(30), 70(8). & $2204(\mathrm{C} \equiv \mathrm{N})$ & $\begin{array}{l}2.30-2.11(4 \mathrm{H}, \mathrm{m}, 3,4-\mathrm{H} \text { of pyrrolidinyl }), 2.82(3 \mathrm{H}, \mathrm{s}, \\
\left.\mathrm{CH}_{3}\right), 4.09(2 \mathrm{H}, \mathrm{t}, J=1.40 \mathrm{~Hz}, 5-\mathrm{H} \text { of pyrrolidinyl }), 4.21 \\
(2 \mathrm{H}, \mathrm{t}, J=1.40 \mathrm{~Hz}, 2-\mathrm{H} \text { of pyrrolidinyl }), 8.08-8.07, \\
7.76-7.57(5 \mathrm{H}, \mathrm{m}, \text { phenyl-H). }\end{array}$ \\
\hline $4 b$ & $\begin{array}{l}\text { 278(100), 263(7), 249(92), 236(26), 223(24), } \\
210(8), 196(12), 154(30), 104(42), 77(17) \\
55(3) .\end{array}$ & $2208(\mathrm{C} \equiv \mathrm{N})$ & $\begin{array}{l}1.76,4.02\left(10 \mathrm{H}, \mathrm{m}, \text { piperidinyl-H), } 2.69\left(3 \mathrm{H}, \mathrm{s}, \mathrm{CH}_{3}\right)\right. \\
8.41-8.39,7.50-7.45(5 \mathrm{H}, \mathrm{m}, \text { phenyl-H). }\end{array}$ \\
\hline $5 \mathbf{a}$ & $\begin{array}{l}\text { 280(100), 249(32), 236(17), 223(91), } \\
\text { 194(16), 153(46), 125(4), } 104(37), 77(24), \\
\text { 56(7). }\end{array}$ & $2210(\mathrm{C} \equiv \mathrm{N})$ & $\begin{array}{l}2.69\left(3 \mathrm{H}, \mathrm{s}, \mathrm{CH}_{3}\right), 3.85(4 \mathrm{H}, \mathrm{d}, J=1.0 \mathrm{~Hz}, 2,6-\mathrm{H} \text { of } \\
\text { morpholinyl), } 4.07(4 \mathrm{H}, \mathrm{d}, J=1.0 \mathrm{~Hz}, 3,5-\mathrm{H} \text { of } \\
\text { morpholinyl), 8.40-8.38, 7.52-7.45 (5H, m, phenyl-H). }\end{array}$ \\
\hline $5 \mathbf{b}$ & $\begin{array}{l}\text { 293(9), 249(4), 236(19), 223(100), 194(4), } \\
\text { 153(11), 104(14), 83(46), 70(43), 55(7). }\end{array}$ & $2205(\mathrm{C} \equiv \mathrm{N})$ & $\begin{array}{l}2.43\left(3 \mathrm{H}, \mathrm{s}, \mathrm{CH}_{3}\right), 2.68\left(3 \mathrm{H}, \mathrm{s}, \mathrm{N}-\mathrm{CH}_{3}\right), 2.66(4 \mathrm{H}, \mathrm{d}, J= \\
1.0 \mathrm{~Hz}, 2,6-\mathrm{H} \text { of piperazinyl }), 4.14(4 \mathrm{H}, \mathrm{d}, J=1.0 \mathrm{~Hz} \\
3,5-\mathrm{H} \text { of piperazinyl), } 8.40-8.38,7.50-7.45(5 \mathrm{H}, \mathrm{m}, \\
\text { phenyl-H). }\end{array}$ \\
\hline $5 c$ & $\begin{array}{l}\text { 307(12), 263(4), 249(5), 236(29), 223(65), } \\
\text { 194(4), 153(11), 104(12), 97(60), 84(100), } \\
\text { 72(14), 55(7). }\end{array}$ & $2206(\mathrm{C} \equiv \mathrm{N})$ & $\begin{array}{l}1.14\left(3 \mathrm{H}, \mathrm{t}, J=1.44 \mathrm{~Hz}, \mathrm{~N}-\mathrm{CH}_{2} \mathrm{CH}_{3}\right), 2.67\left(3 \mathrm{H}, \mathrm{s}, \mathrm{CH}_{3}\right), \\
2.51\left(2 \mathrm{H}, \mathrm{q}, J=2.16 \mathrm{~Hz}, \mathrm{~N}-\mathrm{CH}_{2} \mathrm{CH} 3\right), 2.62(4 \mathrm{H}, \mathrm{d}, J= \\
1.0 \mathrm{~Hz}, 2,6-\mathrm{H} \text { of piperazinyl }), 4.11(4 \mathrm{H}, \mathrm{d}, J=1.0 \mathrm{~Hz}, \\
3,5-\mathrm{H} \text { of piperazinyl), 8.40-8.38, 7.50-7.44 }(5 \mathrm{H}, \mathrm{m}, \\
\text { phenyl-H). }\end{array}$ \\
\hline $5 d$ & $\begin{array}{l}351(95), 336(1), 322(8), 306(2), 283(17), \\
278(2), 249(9), 236(382), 223(100), 194(6), \\
153(1), 141(10), 58(4) .\end{array}$ & $\begin{array}{l}1705(\mathrm{C}=\mathrm{O}) \\
2207(\mathrm{C} \equiv \mathrm{N})\end{array}$ & $\begin{array}{l}1.30\left(3 \mathrm{H}, \mathrm{t}, J=1.43 \mathrm{~Hz}, \mathrm{OCH}_{2} \mathrm{CH}_{3}\right), 2.69\left(3 \mathrm{H}, \mathrm{s}, \mathrm{CH}_{3}\right) \text {, } \\
3.67(4 \mathrm{H}, \mathrm{t}, J=1.06 \mathrm{~Hz}, 2,6-\mathrm{H} \text { of piperazinyl }), 4.05(4 \mathrm{H} \text {, } \\
\mathrm{d}, J=1.00 \mathrm{~Hz}, 3,5-\mathrm{H} \text { of piperazinyl }), 4.20(2 \mathrm{H}, \mathrm{q}, J= \\
2.13 \mathrm{~Hz}, \mathrm{COOCH}), 8.40-8.38,7.53-7.45(5 \mathrm{H}, \mathrm{m}, \\
\text { phenyl-H). }\end{array}$ \\
\hline $5 e$ & $\begin{array}{l}355(42), 249(5), 236(28), 223(100), 194(3), \\
\text { 177(4), 153(10), 132(94), 120(31), 104(44), } \\
77(20), 55(5) .\end{array}$ & $2200(\mathrm{C} \equiv \mathrm{N})$ & $\begin{array}{l}2.70\left(3 \mathrm{H}, \mathrm{s}, \mathrm{CH}_{3}\right), 3.37(4 \mathrm{H}, \mathrm{d}, J=1.09 \mathrm{~Hz}, 2,6-\mathrm{H} \text { of } \\
\text { piperazinyl }), 4.25(4 \mathrm{H}, \mathrm{d}, J=1.00 \mathrm{~Hz}, 3,5-\mathrm{H} \text { of } \\
\text { piperazinyl }), 8.43-8.42,7.52-6.91(10 \mathrm{H}, \mathrm{m} \text {, phenyl- } \mathrm{H}) \text {. }\end{array}$ \\
\hline $5 f$ & $\begin{array}{l}357(57), 262(9), 249(15), 236(34), 223(100) \\
\text { 194(5), 179(6), 153(11), 147(44), 134(67), } \\
\text { 123(24), 108(39), 103(16), 80(22), 55(5). }\end{array}$ & $2204(\mathrm{C} \equiv \mathrm{N})$ & $\begin{array}{l}2.69\left(3 \mathrm{H}, \mathrm{s}, \mathrm{CH}_{3}\right), 4.02(4 \mathrm{H}, \mathrm{d}, J=1.06 \mathrm{~Hz}, 2,6-\mathrm{H} \text { of } \\
\text { piperazinyl }), 4.16(4 \mathrm{H}, \mathrm{d}, J=1.06 \mathrm{~Hz}, 3,5-\mathrm{H} \text { of } \\
\text { piperazinyl }), 6.56(1 \mathrm{H}, \mathrm{m}, 5-\mathrm{H} \text { of pyrimidinyl }), 8.36 \\
(2 \mathrm{H}, \mathrm{d}, J=1.0 \mathrm{~Hz}, 4,6-\mathrm{H} \text { of pyrimidinyl }), 8.42-8.40 \text {, } \\
7.51-7.45(5 \mathrm{H}, \mathrm{m} \text {, phenyl-H). }\end{array}$ \\
\hline
\end{tabular}

${ }^{a}$ Abbreviations: $\mathrm{s}$, singlet; $\mathrm{d}$, doublet; $\mathrm{t}$, triplet; q, quartet; $\mathrm{m}$, multiplet.

2.7. 5-Amino-6-benzoyl-4-methyl-2-phenylthieno[2,3-d]pyrimidine (10). A mixture of 5-cyano-1,6-dihydro-4-methyl2-phenyl-6-thioxopyrimidine $1(2.27 \mathrm{~g}, 0.01 \mathrm{~mol})$, 2-bromoacetophenone $9(1.99 \mathrm{~g}, 0.01 \mathrm{~mol})$, and sodium ethoxide $(1.36 \mathrm{~g}, 0.02 \mathrm{~mol})$ in DMF $(50 \mathrm{~mL})$ was stirred at room temperature for $4 \mathrm{~h}$ and then diluted with cold water $(50 \mathrm{~mL})$. The resulting solid product was collected by filtration, washed with water, and recrystallized from DMF/ ethanol to give $3.17 \mathrm{~g}$ of yellow needles (92\% yield), $\mathrm{mp}$ $236^{\circ} \mathrm{C}$; IR: $v$ 3421, $3288\left(\mathrm{NH}_{2}\right), 1663(\mathrm{C}=\mathrm{O}) \mathrm{cm}^{-1}$; ${ }^{1} \mathrm{H}-\mathrm{NMR}$ $\left(\mathrm{DMSO}-d_{6}\right): \delta 2.56\left(3 \mathrm{H}, \mathrm{s}, \mathrm{CH}_{3}\right), 3.71\left(2 \mathrm{H}, \mathrm{br}, \mathrm{NH}_{2}\right)$, 8.39-8.37, 7.95-7.72 (10H, m, phenyl-H); MS $(m / z, \%)$ : 345( $\left.\mathrm{M}^{+}, 100\right)$. Anal. Calcd. for $\mathrm{C}_{20} \mathrm{H}_{15} \mathrm{~N}_{3}$ OS: C, 69.56; $\mathrm{H}$, 4.34 ; N, 12.17. Found: C, 69.71; H, 4.50; N, 12.43\%.
2.8. 6-Benzoyl-4-methyl-5-(1-pyrrolyl)-2-phenylthieno [2,3d]pyrimidine (11). A mixture of 5-amino-6-benzoyl4-methyl-2-phenylthieno[2,3-d]pyrimidine $\mathbf{1 0}$ (0.34 g, $1 \mathrm{mmol})$ and 2,5-dimethoxytetrahydrofuran $(0.13 \mathrm{~g}$, $1 \mathrm{mmol})$, in glacial acetic acid $(20 \mathrm{~mL})$ was refluxed for $12 \mathrm{~h}$. After cooling, the resulting solid product was collected by filtration and washed with water, and the crude product recrystallized from ethanol/glacial acetic acid to give $0.18 \mathrm{~g}$ of brown needles ( $46 \%$ yield), mp $130^{\circ} \mathrm{C}$; IR: $v 1663(\mathrm{C}=\mathrm{O})$ $\mathrm{cm}^{-1} ;{ }^{1} \mathrm{H}-\mathrm{NMR}\left(\mathrm{CDCl}_{3}\right): \delta 2.31\left(3 \mathrm{H}, \mathrm{s}, \mathrm{CH}_{3}\right), 6.10(2 \mathrm{H}, \mathrm{m}$, 3,4-H of pyrrolyl), 6.71 ( $2 \mathrm{H}, \mathrm{m}, 2,5-\mathrm{H}$ of pyrrolyl), 8.59-8.57, 7.69-7.31 (10H, m, phenyl-H); MS ( $m / z, \%): 395\left(\mathrm{M}^{+}, 100\right)$. Anal. Calcd. for $\mathrm{C}_{24} \mathrm{H}_{17} \mathrm{~N}_{3}$ OS: C, 72.91; H, 4.30; N, 10.63. Found: C, 73.01; H, 4.42; N, 10.67\%. 

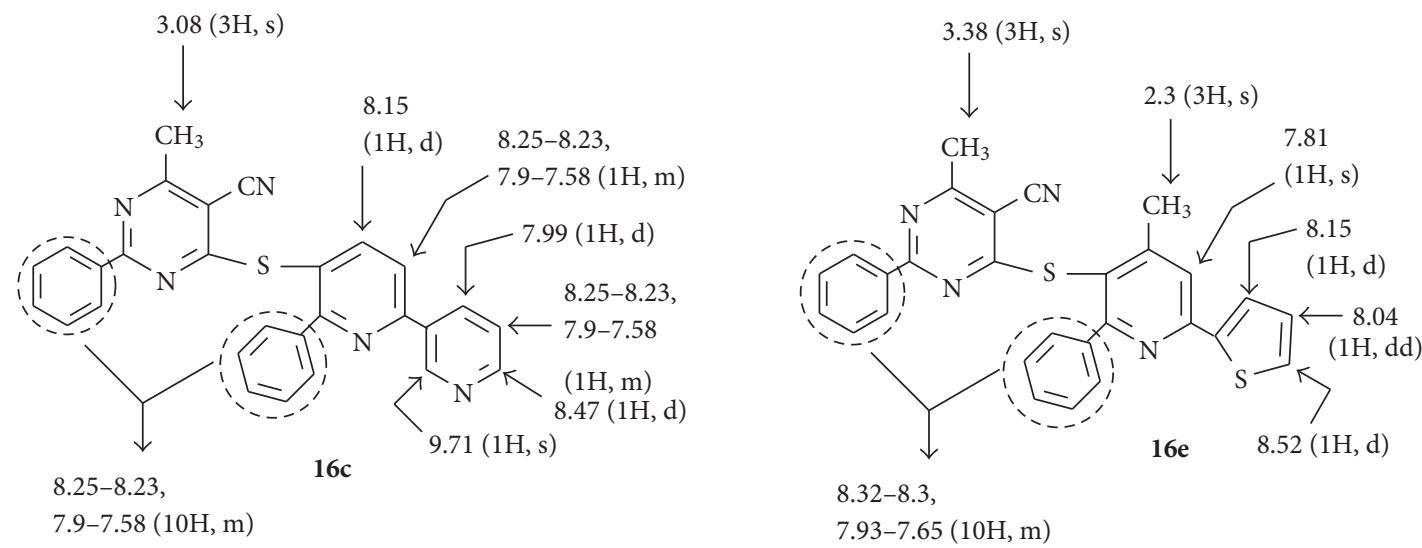

FIGURE 4: Structural assignment of typical protons in $16 \mathrm{c}$ and $16 \mathrm{e}$ by ${ }^{1} \mathrm{H}$ NMR.<smiles>[Y][Z]([H])([H])c1ccc(-c2nc(C)c(C#N)c(N3CCC3)n2)cc1</smiles>

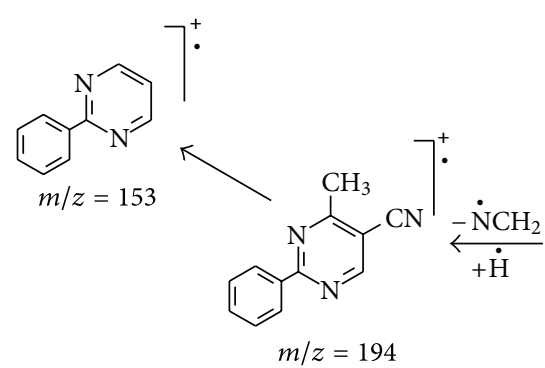

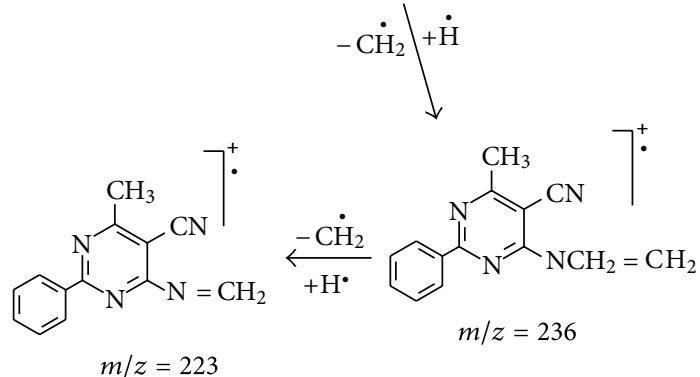

Figure 5

2.9. 5-(1,2,3,4-Tetrazol-1-yl)-6-benzoly-4-methyl-2-phenylthieno[2,3-d]pyrimidine (12). A mixture of 5-amino-6benzoyl-4-methyl-2-phenylthieno[2,3-d]pyrimidine 10 $(0.34 \mathrm{~g}, 1 \mathrm{mmol})$, sodium azide $(0.065 \mathrm{~g}, 1 \mathrm{mmol})$, and triethyl orthoformate $(8 \mathrm{~mL})$ was refluxed in acetic anhydride $(15 \mathrm{~mL})$ for $4 \mathrm{~h}$. The reaction mixture was cooled. The resulting solid product was collected by filtration and washed with water, and the crude product recrystallized from DMF/glacial acetic acid to give $0.31 \mathrm{~g}$ of orange yellow needles (78\% yield), mp $196^{\circ} \mathrm{C}$; IR: $v 1662(\mathrm{C}=\mathrm{O}) \mathrm{cm}^{-1}$; ${ }^{1} \mathrm{H}-\mathrm{NMR}\left(\mathrm{CF}_{3} \mathrm{COOD}\right): \delta 2.61\left(3 \mathrm{H}, \mathrm{s}, \mathrm{CH}_{3}\right), 8.83(1 \mathrm{H}, \mathrm{s}, 5-\mathrm{H}$ of tetrazoly), 8.64-8.62, 8.21-7.96 (10H, $\mathrm{m}$, phenyl-H); MS $(m / z, \%): 398\left(\mathrm{M}^{+}, 10\right), 367(13), 344(100), 268(3), 240(10)$, 211(2), 105(5). Anal. Calcd. for $\mathrm{C}_{21} \mathrm{H}_{14} \mathrm{~N}_{6} \mathrm{OS}$ : C, 63.31; $\mathrm{H}$, $3.51 ; \mathrm{N}, 21.10$. Found: C, 63.45; H, 3.66; N, 21.31\%.

2.10. 6-[(Benzoylmethyl)thio]-5-cyano-4-methyl-2-phenyl-pyrimidine (13). A mixture of 5-cyano-1,6-dihydro-4-methyl2-phenyl-6-thioxopyrimidine $\mathbf{1}(2.27 \mathrm{~g}, 0.01 \mathrm{~mol})$, sodium ethoxide $(0.68 \mathrm{~g}, 0.01 \mathrm{~mol})$, and 2-bromoacetophenone 9
$(1.99 \mathrm{~g}, 0.01 \mathrm{~mol})$ in DMF $(50 \mathrm{ml})$. The reaction mixture was stirred at room temperature for $4 \mathrm{~h}$ and then diluted with cold water $(50 \mathrm{~mL})$ was collected by filtration, washed with water, and recrystallized from chlorofrom/ethanol to give $3.1 \mathrm{~g}$ of pale yellow needles ( $90 \%$ yield), mp $169^{\circ} \mathrm{C}$; IR: $v$ 2220(C $\equiv \mathrm{N}), 1679(\mathrm{C}=\mathrm{O}) \mathrm{cm}^{-1} ;{ }^{1} \mathrm{H}-\mathrm{NMR}\left(\mathrm{CDCl}_{3}\right): \delta 2.67$ $\left(3 \mathrm{H}, \mathrm{s}, \mathrm{CH}_{3}\right), 4.77\left(2 \mathrm{H}, \mathrm{s}, \mathrm{SCH}_{2}\right), 8.11-8.06,7.70-7.22(10 \mathrm{H}$, $\mathrm{m}$, phenyl-H); MS ( $\mathrm{m} / z, \%): 345\left(\mathrm{M}^{+}, 85\right), 319(15), 312(30)$, 242(3), 240(30), 134(10), 105(100), 77(4). Anal. Calcd. for $\mathrm{C}_{20} \mathrm{H}_{15} \mathrm{~N}_{3}$ OS: C, 69.56; H, 4.34; N, 12.17. Found: C, 69.68; $\mathrm{H}, 4.40 ; \mathrm{N}, 12.33 \%$.

2.11. 6-[(4,5,6-Trisubstituted-2-phenyl-pyridin-3-yl)thio]5-cyano-4-methyl-2-phenyl-pyrimidine (16a-f) General Procedure. A mixture of compound $13(0.35 \mathrm{~g}, 1 \mathrm{mmol})$ and enaminone derivatives $14 \mathbf{a}-\mathbf{f}(1 \mathrm{mmol})$ and ammonium acetate $(2 \mathrm{mmol})$ was refluxed in glacial acetic acid $(10 \mathrm{~mL})$ for $11 \mathrm{~h}$. After cooling, the resulting solid product was collected by filtration, washed with water, and recrystallized from ethanol/glacial acetic acid. The physical constants and 
<smiles>Cc1nc(-c2ccccc2)nc(Sc2ccc(-c3cccnc3)nc2-c2ccccc2)c1C#N</smiles>

$m / z=457$<smiles>C[Te]C[Te]</smiles>

$m / z=430$<smiles>C[C+]CCCCCC</smiles>

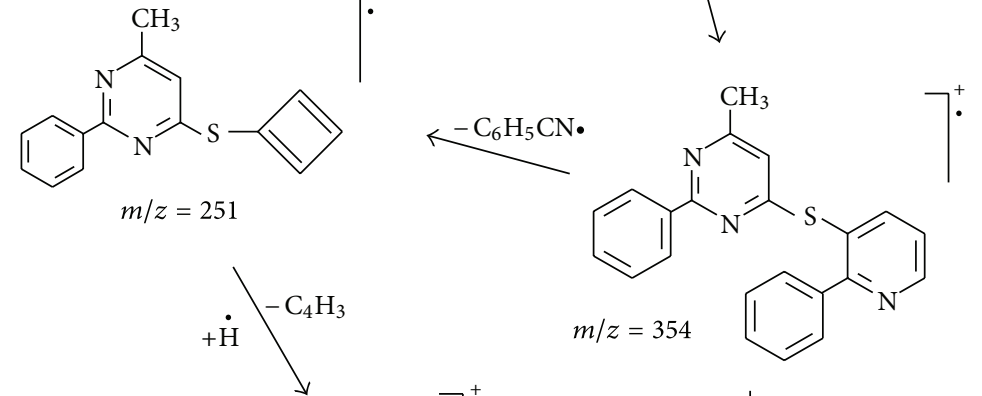<smiles>Sc1ccnc(-c2ccccc2)n1</smiles>

$m / z=186$<smiles>CCCCCCCC</smiles><smiles>c1ccc([N+]23CCCCC2C3)cc1</smiles>

Figure 6

spectral data of compounds $\mathbf{1 6} \mathbf{a}-\mathbf{f}$ are recorded in Tables 6 and 7.

2.12. 2-[(5-Cyano-4-methyl-2-phenylpyrimidin-6-yl)thio]-3dimethylamino-1-phenyl-prop-2-en-1-one (17). A mixture of compound 13 (0.35 g, $1 \mathrm{mmol})$ and N,N-dimethylformamide dimethylacetal $(1 \mathrm{mmol})$ was refluxed in xylene $(5 \mathrm{~mL})$ for $5 \mathrm{~h}$. After cooling, the resulting solid product was collected by filtration, washed with water, and recrystallized from ethanol/glacial acetic acid afforded $0.26 \mathrm{~g}$ of yellow crystals (66\% yield), mp $185^{\circ} \mathrm{C}$; IR: $v 2214(\mathrm{C} \equiv \mathrm{N}), 1654(\mathrm{C}=\mathrm{O}) \mathrm{cm}^{-1}$; ${ }^{1} \mathrm{H}-\mathrm{NMR}\left(\mathrm{CF}_{3} \mathrm{COOD}\right): \delta 2.94\left(6 \mathrm{H}, \mathrm{s}, \mathrm{N}\left(\mathrm{CH}_{3}\right)\right), 3.43(3 \mathrm{H}$, s, $\left.\mathrm{CH}_{3}\right), 7.47(1 \mathrm{H}, \mathrm{s},=\mathrm{CH}-\mathrm{N}), 8.27-8.23,7.81-7.57(10 \mathrm{H}$, $\mathrm{m}$, phenyl-H); MS (m/z, \%): 400( $\left.\mathrm{M}^{+}, 10\right), 344(100)$. Anal. Calcd. for $\mathrm{C}_{23} \mathrm{H}_{20} \mathrm{~N}_{4} \mathrm{OS}$ : C,69.00; H, 5.00; N, 14.00. Found: C, 59.93; H, 5.23.; N, 14.33\%.

2.13. 6-[(4-Phenyl-pyrimidin-5-yl)thio]-5-cyano-4-methyl-2phenyl-pyrimidine (21). A mixture of compound 17 (0.40 g, $1 \mathrm{mmol}$ ) and formamide/formic acid (1:1,1 mmol) 20and ammonium acetate $(2 \mathrm{mmol})$ was refluxed for $7 \mathrm{~h}$. After cooling, the resulting solid product was collected by filtration, washed with water, and recrystallized from ethanol/glacial acetic acid to give $0.21 \mathrm{~g}$ of yellow needles ( $56 \%$ yield), mp $258^{\circ} \mathrm{C}$; IR: $v 2209(\mathrm{C} \equiv \mathrm{N}) \mathrm{cm}^{-1}{ }^{1}{ }^{1} \mathrm{H}-\mathrm{NMR}\left(\mathrm{CF}_{3} \mathrm{COOD}\right): \delta 3.84$ $\left(3 \mathrm{H}, \mathrm{s}, \mathrm{CH}_{3}\right), 8.21(1 \mathrm{H}, \mathrm{s}, 6-\mathrm{H}$ of pyrimidinyl), $8.26(1 \mathrm{H}, \mathrm{s}$, 2-H of pyrimidinyl), 8.70-8.69, 8.24-8.02 (10H, m, phenyl$\mathrm{H})$; MS ( $m / z, \%): 381(54), 345(100), 280(16), 256(8), 210(1)$, 121(2), 105(22), 98(6), 57(4). Anal. Calcd. for $\mathrm{C}_{22} \mathrm{H}_{15} \mathrm{~N}_{5} \mathrm{~S}: \mathrm{C}$, 69.29; H, 3.93; N, 18.37. Found: C, 69.38; H, 4.01; N, $18.44 \%$.

2.14. 6-[(Substituted)thio]-5-cyano-4-methyl-2-phenylpyri-midine Derivatives (19, 23, 25, 27 and 29) General Procedure. A mixture of compound $17(0.40 \mathrm{~g}, 1 \mathrm{mmol})$ and phenylhydrazine 18, 2-amino-benzimidazole 22, 5-amino$1 \mathrm{H}$-tetrazole 24, and 5-amino-pyrazoles 26, 28 (1 mmol) in the presence of glacial acetic acid $(5 \mathrm{~mL})$ was refluxed for $7 \mathrm{~h}$. After cooling, the resulting solid product was collected by filtration, washed with water, and the crude product recrystallized from DMF/glacial acetic acid.

2.15. 6-[(1,5-Diphenyl-1h-Pyrazol-4-yl)thio]-5-cyano-4-methyl-2-phenyl-pyrimidine (19). Yield 38\%, mp $240^{\circ} \mathrm{C}$; IR: $v$ $2201(\mathrm{C} \equiv \mathrm{N}) \mathrm{cm}^{-1} ;{ }^{1} \mathrm{H}-\mathrm{NMR}\left(\mathrm{CF}_{3} \mathrm{COOD}\right): \delta 3.34(3 \mathrm{H}, \mathrm{s}$, $\left.\mathrm{CH}_{3}\right), 7.76(1 \mathrm{H}, \mathrm{s}, 3-\mathrm{H}$ of pyrazole), 8.25-8.23, 7.81-7.79, 7.70-7.57 (15H, m, phenyl-H); MS (m/z, \%): 445( $\left.\mathrm{M}^{+}, 7\right)$, 422(6), 355 (4), 344(100), 268(3), 93(2). Anal. Calcd. for $\mathrm{C}_{27} \mathrm{H}_{19} \mathrm{~N}_{5} \mathrm{~S}$ : C, 72.80; H, 4.26; N, 15.73. Found: C, 72.91; H, 4.47 ; N, $15.89 \%$. 
TABLE 3: Crystal data and structure refinement for compound 13.

\begin{tabular}{|c|c|}
\hline Empirical formula & $\mathrm{C}_{20} \mathrm{H}_{15} \mathrm{~N}_{3} \mathrm{OS}$ \\
\hline Formula weight & 345.41 \\
\hline Temperature & 297(2) K \\
\hline Wavelength & $0.71073 \AA$ \\
\hline Crystal system & Monoclinic \\
\hline Space group & $\mathrm{P} 21 / \mathrm{n}$ \\
\hline \multirow{6}{*}{ Unit cell dimensions } & $a=13.5314(9) \AA$ \\
\hline & $\alpha=90^{\circ}$ \\
\hline & $b=9.5316(6) \AA$ \\
\hline & $\beta=110.7820(10)^{\circ}$. \\
\hline & $c=14.7941(10) \AA$ \\
\hline & $\gamma=90^{\circ}$ \\
\hline Volume & $1783.9(2) \AA^{3}$ \\
\hline$Z$ & 4 \\
\hline Density (calculated) & $1.286 \mathrm{Mg} / \mathrm{m}^{3}$ \\
\hline Absorption coefficient & $0.193 \mathrm{~mm}^{-1}$ \\
\hline $\mathrm{F}(000)$ & 720 \\
\hline Crystal size & $0.67 \times 0.63 \times 0.46 \mathrm{~mm}^{3}$ \\
\hline Theta range for data collection & 2.60 to $26.01^{\circ}$. \\
\hline Index ranges & $\begin{array}{l}-16 \leq h \leq 16,-11 \leq k \leq 11 \\
-11 \leq l \leq 18\end{array}$ \\
\hline Reflections collected & 9753 \\
\hline Independent reflections & $3499[R(\mathrm{int})=0.0305]$ \\
\hline Completeness to theta $=26.01^{\circ}$ & $99.7 \%$ \\
\hline Absorption correction & Empirical \\
\hline Max. and min. transmission & 0.9163 and 0.8813 \\
\hline Refinement method & Full-matrix least-squares on $F^{2}$ \\
\hline Data/restraints/parameters & $3499 / 0 / 226$ \\
\hline Goodness-of-fit on $F^{2}$ & 1.016 \\
\hline Final $R$ indices $[\mathrm{I}>2 \operatorname{sigma}(\mathrm{I})]$ & $R_{1}=0.0478, w R_{2}=0.1350$ \\
\hline$R$ indices (all data) & $R_{1}=0.0587, w R_{2}=0.1474$ \\
\hline Largest diff. peak and hole & 0.322 and $-0.391 \mathrm{e} \cdot \AA^{-3}$ \\
\hline
\end{tabular}

2.16. 6-[(4-Phenyl-benzimidazolo[1,2-a]pyrimidinyl-3-yl) thio]-5-cyano-4-methyl-2- phenyl-pyrimidine (23). Yield $54 \%, \mathrm{mp} 232^{\circ} \mathrm{C}$; IR: $v 2204(\mathrm{C} \equiv \mathrm{N})(\mathrm{C}=\mathrm{N}) \mathrm{cm}^{-1} ;{ }^{1} \mathrm{H}-\mathrm{NMR}$ $\left(\mathrm{CF}_{3} \mathrm{COOD}\right): \delta 2.51\left(3 \mathrm{H}, \mathrm{s}, \mathrm{CH}_{3}\right), 8.53(1 \mathrm{H}, \mathrm{s}, 2-\mathrm{H}$ of benzimidazolo- pyrimidinyl), 8.09-8.08, 7.98-7.86 $(14 \mathrm{H}, \mathrm{m}$, benzimidazolyl-H and phenyl-H); MS (m/z, \%): 470(11), 430(2), 367(2), 355(30), 345(100), 276(2), 227(8), 105(4). Anal. Calcd. for $\mathrm{C}_{28} \mathrm{H}_{18} \mathrm{~N}_{6} \mathrm{~S}$ : C, 71.48; H, 3.82; N, 17.87 . Found: C, 71.39; H, 3.99; N, 17.98\%.

\subsection{6-[(4-Phenyl-1,2,3,4-tetrazolo[1,5-a]pyrimidinyl-} 3-yl)thio]-5-cyano-4-methyl-2-phenyl-pyrimidine (25). Yield 50\%, mp $233^{\circ} \mathrm{C}$; IR: $v 2206(\mathrm{C} \equiv \mathrm{N}) \mathrm{cm}^{-1} ;{ }^{1} \mathrm{H}-\mathrm{NMR}$ $\left(\mathrm{CF}_{3} \mathrm{COOD}\right): \delta 2.63\left(3 \mathrm{H}, \mathrm{s}, \mathrm{CH}_{3}\right), 8.65(1 \mathrm{H}, \mathrm{s}, 2-\mathrm{H}$ of tetrazolopyrimidinyl), 8.22-8.17, 8.11-7.99 $(10 \mathrm{H}, \mathrm{m}$, phenyl-H); MS (m/z, \%): 422(6), 344(100), 292(2), 212(5), 105(3). Anal. Calcd. for $\mathrm{C}_{22} \mathrm{H}_{14} \mathrm{~N}_{8} \mathrm{~S}$ : C, 62.55; H, 3.32; N, 26.54. Found: C, 62.74; H, 3.45; N, 26.66\%.
2.18. 6-[(5-Cyano-6-methyl-2-phenylpyrimidin-4-yl)thio]2-methyl-3-phenylazo-7-phenyl-pyrazolo[1,5-a]pyrimidine (27). Yield 67\%, mp $230^{\circ} \mathrm{C}$; IR: $v 2200(\mathrm{C} \equiv \mathrm{N}) \mathrm{cm}^{-1}$; ${ }^{1} \mathrm{H}-\mathrm{NMR}\left(\mathrm{CF}_{3} \mathrm{COOD}\right): \delta 2.62\left(3 \mathrm{H}, \mathrm{s}, \mathrm{CH}_{3}\right), 2.91(3 \mathrm{H}, \mathrm{s}$, $\left.\mathrm{CH}_{3}\right), 8.00(1 \mathrm{H}, \mathrm{s}, 5-\mathrm{H}$ of pyrazolopyrimidinyl), 8.28-8.27, 7.84-7.61 (15H, m, phenyl-H); MS ( $m / z, \%): 538(15)$, 461(8), 344(100), 316(10), 240(4), 182(1), 124(1), 93(2). Anal. Calcd. for $\mathrm{C}_{31} \mathrm{H}_{22} \mathrm{~N}_{8} \mathrm{~S}$ : C, 69.14; H, 4.08; N, 20.81 . Found: C, 69.26; H, 4.22; N, 20.66\%.

\subsection{7-[(5-Cyano-6-methyl-2-phenylpyrimidin-4-yl)thio]- 4-methyl-2,8-diphenyl-pyrimido[4, 5 :3,4]pyrazolo[1,5- a]pyrimidine (29). Yield 65\%, mp $289^{\circ} \mathrm{C}$; IR: $v 2201(\mathrm{C} \equiv \mathrm{N})$ $\mathrm{cm}^{-1}$; ${ }^{1} \mathrm{H}-\mathrm{NMR}\left(\mathrm{CF}_{3} \mathrm{COOD}\right): \delta 2.37\left(3 \mathrm{H}, \mathrm{s}, \mathrm{CH}_{3}\right), 3.52(3 \mathrm{H}$, s, $\left.\mathrm{CH}_{3}\right), 7.67$ (1H, s, 6- $\mathrm{H}$ of pyrimidopyrazolopyrimidinyl), 8.41-8.39, 7.95-7.72 (15H, m, phenyl-H); MS (m/z, \%): 562(15), 460(1), 418(2), 316(10), 368(100), 344(20), 249(4), 225(16), 197(10), 153(12), 104(13), 77(17), 51(4). Anal. Calcd. for $\mathrm{C}_{33} \mathrm{H}_{22} \mathrm{~N}_{8} \mathrm{~S}$ : C, 70.46; H, 3.91; N, 19.92. Found: C, 70.66; H, 4.12; N, 20.16\%.}

2.20. X-Ray Structure Study of Compound 13. The diffraction data of compound $\mathbf{1 3}$ was collected on a Siemens CCD diffractometer, which was equipped with graphitemonochromated Mo- $\mathrm{K}_{\alpha}\left(\mathrm{K}_{\alpha}=0.71073 \AA\right)$ radiation. Data reduction was carried by standard methods with use of wellestablished computational procedures $[28,29]$. A pale yellow crystal of compound $\mathbf{1 3}$ was mounted on the top of a glass fiber with epoxy cement. The hemisphere data collection method was used to scan the data points at $3.34<2 \theta<$ $52.02^{\circ}$. The structure factors were obtained after Lorentz and polarization correction. The final residuals of the final refinement were $\mathrm{R}_{1}=0.0478, \omega \mathrm{R}_{2}=0.1350$. The crystallographic data of compound 13 has been deposited with the Cambridge Crystallographic Data Center as supplementary publication no. CCDC 752301. Copy of this information may be obtained free of charge via http://www.ccdc.cam.ac.uk or from The Director, CCDC, 12 Union Road, Cambridge CB2 1EZ, UK (fax: +441223/336-033; email: deposit@ccdc.cam.ac.uk).

\section{Results and Discussion}

All relevant reactions are depicted in Schemes 1, 2, 3, and 4. The required compound 5-cyano-1,6-dihydro-4-methyl-2phenyl-6-thioxopyrimidine 1 was prepared by treating benzoylisothiocyanate with 3 -aminocrotononitrile in refluxing dioxane [26]. Several pyrimidines substituted at position6 with different heterocyclic residues were obtained via treatment of thioxopyrimidine 1 with different reagents. Thus, reaction of thioxopyrimidine $\mathbf{1}$ with methyl iodide in the presence of sodium methoxide to yield the 6methylthio-4-methyl-2-phenyl-pyrimidine 2 , which reacted with selenium oxide in dioxane afforded the 4-formyl-6methylthio-2-phenyl-pyrimidine 3 (Scheme 1). The IR spectra of compound 3 showed the characteristic absorption bands at $1689 \mathrm{~cm}^{-1}$ for the $\mathrm{HC}=\mathrm{O}$ group and $2218 \mathrm{~cm}^{-1}$ for 
TABLE 4: Selected bond lengths [Å] and angles $\left[^{\circ}\right]$ for compound $13^{\mathrm{a}}$.

\begin{tabular}{|c|c|}
\hline S-C(10) & $1.743(17)$ \\
\hline $\mathrm{O}-\mathrm{C}(14)$ & $1.209(2)$ \\
\hline $\mathrm{N}(1)-\mathrm{C}(7)$ & $1.341(2)$ \\
\hline $\mathrm{N}(2)-\mathrm{C}(7)$ & $1.339(2)$ \\
\hline$C(1)-C(6)$ & $1.383(3)$ \\
\hline$C(6)-C(7)$ & $1.481(2)$ \\
\hline $\mathrm{C}(9)-\mathrm{C}(10)$ & $1.401(2)$ \\
\hline $\mathrm{N}(6)-\mathrm{C}(12)$ & $1.423(3)$ \\
\hline$C(14)-C(15)$ & $1.482(3)$ \\
\hline$C(10)-S-C(13)$ & $100.0(9)$ \\
\hline $\mathrm{C}(10)-\mathrm{N}(2)-\mathrm{C}(7)$ & $117.0(13)$ \\
\hline $\mathrm{N}(1)-\mathrm{C}(7)-\mathrm{C}(6)$ & $117.3(15)$ \\
\hline $\mathrm{C}(8)-\mathrm{C}(9)-\mathrm{C}(10)$ & $118.0(14)$ \\
\hline $\mathrm{N}(2)-\mathrm{C}(10)-\mathrm{S}$ & $119.6(12)$ \\
\hline $\mathrm{O}-\mathrm{C}(14)-\mathrm{C}(15)$ & $122.1(19)$ \\
\hline$C(15)-C(14)-C(13)$ & $116.3(17)$ \\
\hline$C(16)-C(15)-C(14)$ & $122.8(2)$ \\
\hline S-C(13) & $1.784(2)$ \\
\hline $\mathrm{N}(1)-\mathrm{C}(8)$ & $1.335(2)$ \\
\hline $\mathrm{N}(2)-\mathrm{C}(10)$ & $1.334(19)$ \\
\hline $\mathrm{N}(3)-\mathrm{C}(12)$ & $1.135(3)$ \\
\hline$C(5)-C(6)$ & $1.389(3)$ \\
\hline$C(8)-C(9)$ & $1.387(3)$ \\
\hline$C(13)-C(14)$ & $1.523(3)$ \\
\hline$C(13)-C(14)$ & $1.349(5)$ \\
\hline$C(15)-C(16)$ & $1.393(3)$ \\
\hline $\mathrm{C}(8)-\mathrm{N}(1)-\mathrm{C}(7)$ & $117.4(15)$ \\
\hline $\mathrm{N}(1)-\mathrm{C}(7)-\mathrm{N}(2)$ & $125.7(14)$ \\
\hline $\mathrm{N}(2)-\mathrm{C}(7)-\mathrm{C}(6)$ & $116.8(14)$ \\
\hline $\mathrm{N}(2)-\mathrm{C}(10)-\mathrm{C}(9)$ & $120.9(15)$ \\
\hline$C(9)-C(10)-S$ & $119.3(12)$ \\
\hline $\mathrm{O}-\mathrm{C}(14)-\mathrm{C}(13)$ & 121.4(19) \\
\hline$C(16)-C(15)-C(20)$ & $118.6(2)$ \\
\hline$C(20)-C(15)-C(14)$ & $118.6(2)$ \\
\hline
\end{tabular}

${ }^{\mathrm{a}}$ Standard deviations in parentheses.

the $\mathrm{C} \equiv \mathrm{N}$ group. In addition, the ${ }^{1} \mathrm{H}$ NMR spectra (DMSO$\left.d_{6}\right)$ of compound 3 revealed two singlets at $\delta 2.74(3 \mathrm{H}$, s) and $9.85(1 \mathrm{H}, \mathrm{s})$, which were readily assigned to the $\mathrm{SCH}_{3}$ and $\mathrm{HC}=\mathrm{O}$ groups, respectively. On the other hand, a series of novel 6-substituted-pyrimidine derivatives $\mathbf{4 a , b}$ and $\mathbf{5 a}-\mathbf{f}$ were also obtained by the condensation reaction of compound 2 with appropriate secondary amines such as pyrrolidine, piperidine, morpholine, $\mathrm{N}$-methylpiperazine, $\mathrm{N}$-ethylpiperazine, ethyl 1-piperazinecarboxylate, 1-phenylpiperazine and 1-(2-pyrimidyl)piperazine, (Scheme 1). Compounds $\mathbf{4 a}, \mathbf{b}$ and $\mathbf{5 a}-\mathbf{f}$ were obtained generally in $28-89 \%$ yields. The structures of $\mathbf{4} \mathbf{a}, \mathbf{b}$ and $\mathbf{5 a}-\mathbf{f}$ were verified by elemental analysis and by spectroscopic methods. Physical and spectral data of compounds $\mathbf{4} \mathbf{a}, \mathbf{b}$ and $\mathbf{5 a}-\mathbf{f}$ are recorded in Tables 1 and 2, respectively. Typical assignments for $\mathbf{5 f}$ by
${ }^{1} \mathrm{H}-\mathrm{NMR}$ are shown in Figure 1. These structures get further support from mass spectroscopy. The mass fragmentation pattern of compound $\mathbf{5 d}$ showed the presence of the ion peaks $\left[\mathrm{M}-\mathrm{CH}_{3}\right]^{+}$at $m / z 336,\left[\mathrm{M}-\mathrm{CH}_{2} \mathrm{CH}_{3}\right]^{+}$at $m / z 322$, $\left[\mathrm{M}-\mathrm{OCH}_{2} \mathrm{CH}_{3}\right]^{+}$at $m / z 306$ and $\left[\mathrm{M}-\mathrm{COOCH}_{2} \mathrm{CH}_{3}\right]^{+}$at $\mathrm{m} / z$ 278. Also, it has been observed that electron impact (EI) spectral has many common features. Compounds $\mathbf{5 a}-\mathbf{f}$ exhibited $m / z 249, m / z 236, m / z 223, m / z 194$ and $m / z 153$ piece peaks. The possible mass fragmentation pathways of compounds 5a-f are shown in Figure 5.

Next, treatment of 6-piperidinyl-4-methyl-pyrimidine 4b with $\mathrm{N}, \mathrm{N}$-dimethyl-aminobenzaldehyde in refluxing dioxane in the presence of catalytic amount of piperidine yielded the 4-[(4-N,N-dimethylamino)-2-phenylvinyl)]-6piperidinyl-5-cyano-2-phenyl-pyrimidine 6 . The ${ }^{1} \mathrm{H}-\mathrm{NMR}$ spectra $\left(\mathrm{CDCl}_{3}\right)$ of compound $\mathbf{6}$ revealed a sharp singlet at $\delta 3.09(6 \mathrm{H}, \mathrm{s})$ assigned to the $-\mathrm{N}\left(\mathrm{CH}_{3}\right)_{2}$ protons and at $\delta$ $6.70(1 \mathrm{H}, \mathrm{d})$ and $7.74(1 \mathrm{H}, \mathrm{d})$ assigned to the $-\mathrm{CH}=\mathrm{CH}-$ of 4-dimethylaminophenylethylene moiety, two multiplets at $\delta 3.07-2.96,1.75-1.62(10 \mathrm{H}, \mathrm{m})$ assigned to the piperidinyl protons and a multiplet at $\delta 8.57-7.44(9 \mathrm{H}, \mathrm{m})$ assigned to the phenyl protons, were also confirmed by the mass spectrum $\mathrm{m} / z$ 409 $\left(\mathrm{M}^{+}\right)$. However, reaction of compound 2 with benzhydrazide did not produce the desired compound 7 , but led only to the recovery of starting material. On the other hand, the 6-cyanopropylthio-pyrimidine $\mathbf{8}$ was also obtained by treatment of compound $\mathbf{1}$ with 4-chlorobutyronitrile in DMF at room temperature in the presence of sodium ethoxide in a molar ratio of $1: 2$ (Scheme 2). Nevertheless, under same reaction conditions, treatment of compound $\mathbf{1}$ with 2-bromoacetophenone 9 formed the nonisolable S-alkylated intermediate, which via nucleophilic substitution and intramolecular cyclocondensation afforded the corresponding 5-amino6-benzoly-4-methyl-2-phenylthieno[2,3-d]pyrimidine 10, which when reacted with 2,5-dimethoxy-tetrahydrofuran in glacial acetic acid produced the 5-(1-pyrrolyl)-6-benzoly-4methyl-2-phenylthieno[2,3-d]pyrimidine 11. Also, reaction of compound $\mathbf{1 0}$ with sodium azide and triethyl orthoformate in acetic anhydride afforded the corresponding 5-(1,2,3,4tetrazol-1-yl)-6-benzoly-2-phenyl-thieno[2,3- $d$ ] pyrimidine 12 (Scheme 2). The structure of compounds 10-12 was established on the basis of their elemental analysis and spectral data. The IR spectra of compounds $\mathbf{1 1}$ and $\mathbf{1 2}$ indicated the complete disappearance of $\mathrm{NH}_{2}$ and showed the characteristic absorption band at $1663-1662 \mathrm{~cm}^{-1}$ for the $\mathrm{C}=\mathrm{O}$ group. The ${ }^{1} \mathrm{H}$-NMR spectra $\left(\mathrm{CDCl}_{3}\right)$ of compound $\mathbf{1 1}$ revealed two multiplets at $\delta 6.10(2 \mathrm{H}, \mathrm{m})$ and $6.71(2 \mathrm{H}, \mathrm{m})$, which were readily assigned to the hydrogen of the pyrrolyl ring. Moreover, compound 12 showed a singlet at $\delta 8.83(1 \mathrm{H}$, s) assigned to the hydrogen attached at $C_{5}$ of the tetrazolyl ring.

On the other hand, treatment of compound 1 with 2bromoacetophenone 9 in the presence of sodium ethoxide in a molar ratio of 1:1 afforded the open-chain product 6(benzoylmethyl)thio-2-phenyl-pyrimidine $\mathbf{1 3}$ in $90 \%$ yield (Scheme 2). The IR spectra of compound 13 indicated the characteristic absorption bands at $1679 \mathrm{~cm}^{-1}$ for the $\mathrm{C}=\mathrm{O}$ 
TABLE 5: Selected torsion angles for compound 13.

\begin{tabular}{lc}
\hline $\mathrm{C}(13)-\mathrm{S}-\mathrm{C}(10)-\mathrm{N}(2)$ & $-16.1^{\circ}$ \\
$\mathrm{C}(13)-\mathrm{S}-\mathrm{C}(10)-\mathrm{C}(9)$ & $165.5^{\circ}$ \\
$\mathrm{C}(1)-\mathrm{C}(6)-\mathrm{C}(7)-\mathrm{N}(2)$ & $-175.1^{\circ}$ \\
$\mathrm{C}(1)-\mathrm{C}(6)-\mathrm{C}(7)-\mathrm{N}(1)$ & $-175.1^{\circ}$ \\
$\mathrm{C}(16)-\mathrm{C}(15)-\mathrm{C}(14)-\mathrm{O}$ & $165.7^{\circ}$ \\
$\mathrm{C}(20)-\mathrm{C}(15)-\mathrm{C}(14)-\mathrm{O}$ & $-14.1^{\circ}$ \\
$\mathrm{C}(13)-\mathrm{C}(14)-\mathrm{C}(15)-\mathrm{C}(16)$ & $-13.2^{\circ}$ \\
$\mathrm{C}(13)-\mathrm{C}(14)-\mathrm{C}(15)-\mathrm{C}(20)$ & $167.0^{\circ}$ \\
$\mathrm{S}-\mathrm{C}(13)-\mathrm{C}(14)-\mathrm{O}$ & $11.3^{\circ}$ \\
$\mathrm{S}-\mathrm{C}(13)-\mathrm{C}(14)-\mathrm{C}(15)$ & $-169.7^{\circ}$ \\
\hline
\end{tabular}

group and $2220 \mathrm{~cm}^{-1}$ for the $\mathrm{C} \equiv \mathrm{N}$ group. In particular, the ${ }^{1} \mathrm{H}-\mathrm{NMR}$ spectrum $\left(\mathrm{CDCl}_{3}\right)$ of compound 13 revealed a singlet at $\delta 4.77(2 \mathrm{H}, \mathrm{s})$ assigned to the $\mathrm{SCH}_{2} \mathrm{CO}$ protons. The structure of $\mathbf{1 3}$ was unambiguously confirmed by X-ray crystallography. The suitable single crystals of compound $\mathbf{1 3}$ were obtained by slow crystallization from chloroform/DMF at room temperature. Perspective view and the numbering of the atoms are depicted in Figure 2. This drawing clearly establishes the structural formula and also shows the conformation of the molecule. The packing diagram (Figure 3) in the solid state by intermolecular hydrogen bonding between $\mathrm{C}-\mathrm{H}$ of the $-\mathrm{S}-\mathrm{CH}_{2}$ group and $\mathrm{C}=\mathrm{O}$ atom $(\mathrm{C}-\mathrm{H}-\mathrm{O}=$ $\left.2.261 \AA, \angle \mathrm{C}-\mathrm{H}-\mathrm{O}=145.5^{\circ}\right)$. The relevant crystallographic date and structure refinement are recorded in Table 3 . The selected bond lengths and bond angles are listed in Table 4. The hydrogen atoms were refined isotropically in idealized positions riding on the atom to which they are attached. The crystal system of compound $\mathbf{1 3}$ is monoclinic, the space group is $\mathrm{P} 21 / \mathrm{n}$ and data was collected in the range 2.60 to $26.01^{\circ}$. Details of the intensity collection are recorded in Table 3.

The basal plane is formed by phenyl(C(15))-carbonylmethylthio(C(14)), carbonylmethylthio-pyrimidine $(\mathrm{C}(10))$, and pyrimidine $(\mathrm{C}(7))$-phenyl $(\mathrm{C}(6))$ atoms, with bond lengths of $1.482(3), 1.7436(17)$, and 1.481(2) A, respectively. The phenyl ring is in the cis $(\mathrm{Z})$ configuration with respect to the $S$ atom and the fused pyrimidine system (Figure 2). Moreover, the fused pyrimidine system is almost planar and due to the effect of carbonylmethyl-S moiety the phenyl group exhibit noticeable quinoid character that is demonstrated by the shortening of the $\mathrm{C}(17)-\mathrm{C}(18)[1.353(5) \AA], C(18)-C(19)$ [1.383(5) $\AA$ ] , C(19)-C(20) [1.385(4) $\mathrm{\AA}]$, and C(16)-C(17) [1.374(3) $\AA$ ] bond lengths compared to the standard $\mathrm{C}_{\mathrm{ar}}-\mathrm{C}_{\mathrm{ar}}$ distance of $1.397(1) \AA$ [30]. In addition, the $C(16)-C(17)$ phenyl and carbonyl group are in a staggered conformation with respect to the $\mathrm{O}$ atom giving rise to angular distortion at $\mathrm{C}(15)$ and $\mathrm{C}(14)[\mathrm{C}(16)-\mathrm{C}(15)-\mathrm{C}(14) \mathrm{C}(20)-\mathrm{C}(15)-\mathrm{C}(14)]$ (Table 4). The S-C(10) [1.7436(17) $\AA$ ] bond is longer than the $\mathrm{C}(6)-\mathrm{C}(7)[1.481(2) \AA]$, which is probably due to electron withdrawing effect of the $S$ atom. Also, the interesting torsion angles which entirely define the molecule conformation are selected and listed in Table 5. Moreover, the $\mathrm{C}(9)-\mathrm{C}(10)$ [1.401(2) $\AA$ ] and $\mathrm{N}(1)-\mathrm{C}(7)$ [1.341(2) $\AA$ ] bonds are longer than the $\mathrm{N}(2)-\mathrm{C}(10)[1.334(19) \AA]$. The $\mathrm{C}(1)-\mathrm{C}(2)$ phenyl ring has a dihedral angle with $\mathrm{N}(2)-\mathrm{C}(10)$ pyrimidine ring of $171.1^{\circ}$, while the carbonylmethylthio's dihedral angle with this pyrimidine is $101.0^{\circ}$. Furthermore, the $\mathrm{C}(16)-\mathrm{C}(17)$ phenyl ring attached to the carbonylmethyl group and makes a dihedral angle of $112.2^{\circ}$ with pyrimidine ring plane and the $\mathrm{C}(16)-\mathrm{C}(17)$ phenyl ring has a dihedral angle with carbonylmethylthio group of $15.7^{\circ}$.

On the other hand, the reaction of compound 13 with enaminone derivatives $\mathbf{1 4 a - f}$ was also investigated. Thus, it has been found that compound 13 with 3-dimethylamino1-(phenyl)prop-2-enone $\mathbf{1 4 a}$ in refluxing glacial acetic acid in the presence of excess ammonium acetate gave a yellow product of molecular formula $\mathrm{C}_{29} \mathrm{H}_{20} \mathrm{~N}_{4} \mathrm{~S}(55 \%$ yield, $\left.\mathrm{mp} 218^{\circ} \mathrm{C}\right)$. Spectroscopic analyses revealed that 6-[(2,6-diphenyl-pyridin-3-yl)thio]-5-cyano-4-methyl-2phenylpyrimidine 16a was obtained (Scheme 3). The IR spectra of the reaction product indicated the absence of the $\mathrm{C}=\mathrm{O}$ group and showed the characteristic absorption band at $2203 \mathrm{~cm}^{-1}$ for the $\mathrm{C} \equiv \mathrm{N}$ group. The ${ }^{1} \mathrm{H}-\mathrm{NMR}$ spectra $\left(\mathrm{CF}_{3} \mathrm{COOD}\right)$ of the reaction product showed additional two doublets at $\delta 8.44(1 \mathrm{H}, \mathrm{d})$ and $8.76(1 \mathrm{H}, \mathrm{d})$ assigned to the hydrogen attached at $\mathrm{C}_{5}$ and $\mathrm{C}_{4}$ of pyridine moiety, respectively, and at $\delta 8.54-8.52,8.19-7.87(15 \mathrm{H}, \mathrm{m})$ assigned to the phenyl protons. The structure of compound 16a was further confirmed by mass spectrum $\left(\mathrm{m} / z 456\left(\mathrm{M}^{+}\right)\right)$. The formation of compound 16a would involve an initial nucleophilic substitution of the exocyclic methylene group in compound $\mathbf{1 3}$ to the activated double bond in enaminone 14a to form the intermediate 15, which then undergoes amination and intramolecular cyclization via loss of water affording the final product 16a. Similarly, treatment of compound 13 with enaminone derivatives $\mathbf{1 4 b}-\mathbf{f}$, under similar reaction conditions, afforded the corresponding 6-[(4,5,6-trisubstituted-2-phenyl-pyridin-3-yl)thio]-5-

cyano-4-methyl-2-phenyl-pyri-midines 16b-f (Scheme 3). Typical assignments for $16 \mathrm{c}$ and $16 \mathrm{e}$ by ${ }^{1} \mathrm{H}-\mathrm{NMR}$ are shown in Figure 4 . The physical constants and spectral data of compounds 16a-f are recorded in Tables 6 and 7. These structures get further support from mass spectroscopy. The possible mass fragmentation pathway of compounds $\mathbf{1 6 c}$ is shown in Figure 6.

Furthermore, treatment of 6-(benzoylmethyl)thiopyrimidine 13 with $\mathrm{N}, \mathrm{N}$-dimethylformamide dimethylacetal (DMFDMA) gave the enaminone derivative 2-[(5-cyano4-methyl-2-phenylpyrimidin-6-yl)thio]-3-dimethylamino1-phenylprop-2-en-1-one 17(Scheme 4). The ${ }^{1} \mathrm{H}$ NMR spectra $\left(\mathrm{CF}_{3} \mathrm{COOD}\right)$ of compound $\mathbf{1 7}$ revealed a sharp singlet at $\delta 2.94(6 \mathrm{H}, \mathrm{s})$ assigned to the $-\mathrm{N}\left(\mathrm{CH}_{3}\right)_{2}$ protons and at $\delta 7.47(1 \mathrm{H}, \mathrm{s})$ assigned to the $-\mathrm{C}=\mathrm{CH}-\mathrm{N}$, was also confirmed by the mass spectrum $m / z 400\left(\mathrm{M}^{+}\right)$. On the other hand, the study was extended to investigate the behavior of enaminone derivative 17 with different nucleophiles like amino compounds with a view to synthesizing various heterocyclic ring systems. Intramolecular cyclization of enaminone derivative $\mathbf{1 7}$ gave different products depending on reaction reagents. Thus, treatment of enaminone derivative $\mathbf{1 7}$ with phenylhydrazine $\mathbf{1 8}$ in the presence 
TABLE 6: Physical and analytical data of 6-[(4,5,6-trisubstituted-2-phenyl-pyridin-3-yl)thio]-5-cyano-4-methyl-2-phenyl-pyrimidine derivatives (16a-f).

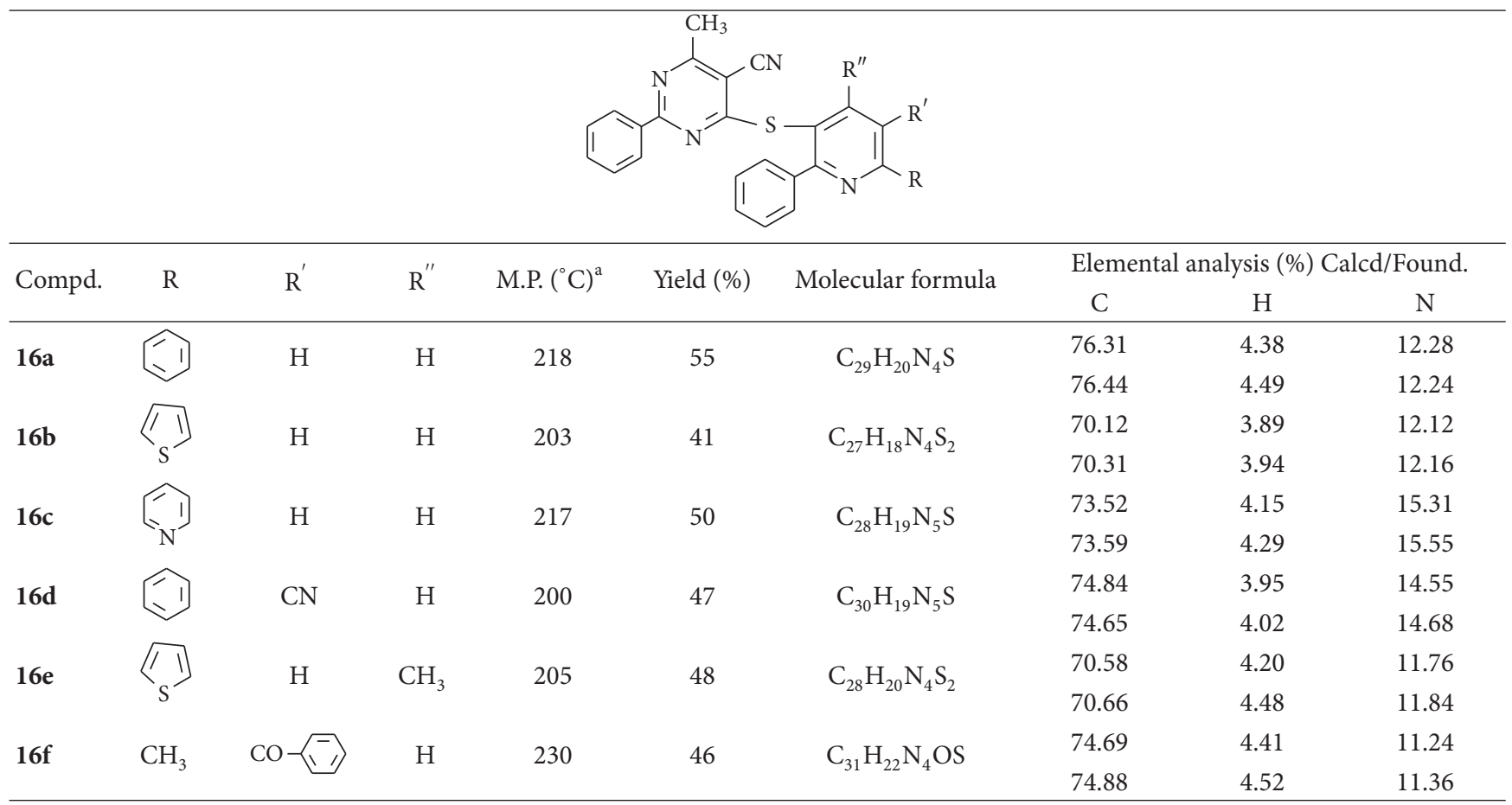

${ }^{\mathrm{a}}$ Recrystallization from $\mathrm{CH}_{3} \mathrm{COOH} / \mathrm{DMF}$.

TABLE 7: Spectral data of 6-[(4,5,6-trisubstituted-2-phenyl-pyridin-3-yl)thio]-5-cyano-4-methyl-2-phenyl-pyrimidine derivatives (16a-f).

\begin{tabular}{|c|c|c|c|}
\hline Compd. & $\mathrm{MS}\left(\mathrm{m} / \mathrm{e} \mathrm{M}^{+}\right)$ & $\begin{array}{l}\operatorname{IR}(\mathrm{KBr}) \\
v\left(\mathrm{~cm}^{-1}\right)\end{array}$ & $\begin{array}{l}{ }^{1} \mathrm{H}-\mathrm{NMR}^{\mathrm{a}} \\
\left(\mathrm{CF}_{3} \mathrm{COOD}\right) \\
\delta(\mathrm{ppm})\end{array}$ \\
\hline $16 a$ & $\begin{array}{l}\text { 456(6), 354(48), 344(100), 328(1), 268(3), } \\
\text { 251(15), 236(1), 186(2), 105(3). }\end{array}$ & $2203(\mathrm{C} \equiv \mathrm{N})$ & $\begin{array}{l}3.67\left(3 \mathrm{H}, \mathrm{s}, \mathrm{CH}_{3}\right), 8.44(1 \mathrm{H}, \mathrm{d}, J=1.01 \mathrm{~Hz}, 5-\mathrm{H} \text { of } \\
\text { pyridyl }), 8.76(1 \mathrm{H}, \mathrm{d}, J=1.01 \mathrm{~Hz}, 4-\mathrm{H} \text { of pyridyl }) \\
8.54-8.52,8.19-7.87(15 \mathrm{H}, \mathrm{m}, \text { phenyl-H). }\end{array}$ \\
\hline $16 b$ & $\begin{array}{l}462(5), 435(10), 430(6), 354(18), 344(100) \\
268(2), 251(6), 185(3), 171(4), 143(7) \\
129(3) .\end{array}$ & $2206(\mathrm{C} \equiv \mathrm{N})$ & $\begin{array}{l}3.63\left(3 \mathrm{H}, \mathrm{s}, \mathrm{CH}_{3}\right), 8.36(1 \mathrm{H}, \mathrm{d}, J=1.00 \mathrm{~Hz}, 5-\mathrm{H} \text { of } \\
\text { pyridyl }), 8.68(1 \mathrm{H}, \mathrm{d}, J=1.00 \mathrm{~Hz}, 4-\mathrm{H} \text { of pyridyl }), 8.20 \\
(1 \mathrm{H}, \mathrm{d}, J=1.00 \mathrm{~Hz}, 3-\mathrm{H} \text { of thienyl-H }), 8.45(1 \mathrm{H}, \mathrm{d}, J= \\
1.00 \mathrm{~Hz}, 5-\mathrm{H} \text { of thienyl-H), } 8.47-8.45,8.13-7.80(11 \mathrm{H} \text {, } \\
\mathrm{m}, 4-\mathrm{H} \text { of thienyl-H and phenyl-H). }\end{array}$ \\
\hline $16 \mathrm{c}$ & $\begin{array}{l}\text { 457(4), 430(9), 368(3), 354(100), 344(90), } \\
\text { 303(8), 268(20), 251(45), 201(3), 186(7), } \\
\text { 105(10). }\end{array}$ & $2208(\mathrm{C} \equiv \mathrm{N})$ & $\begin{array}{l}3.08\left(3 \mathrm{H}, \mathrm{s}, \mathrm{CH}_{3}\right), 7.99(1 \mathrm{H}, \mathrm{d}, J=1.00 \mathrm{~Hz}, 4-\mathrm{H} \text { of } \\
\text { pyridyl), } 8.15(1 \mathrm{H}, \mathrm{d}, J=1.00 \mathrm{~Hz}, 4-\mathrm{H} \text { of S-pyridyl }) \\
8.25-8.23,7.90-7.58(12 \mathrm{H}, \mathrm{m}, 5-\mathrm{H} \text { of } \mathrm{S}-\text { pyridyl, } 5-\mathrm{H} \text { of } \\
\text { pyridyl and phenyl-H), } 8.47(1 \mathrm{H}, \mathrm{d}, J=1.00 \mathrm{~Hz}, 6-\mathrm{H} \text { of } \\
\text { pyridyl), } 9.79(1 \mathrm{H}, \mathrm{s}, 2-\mathrm{H} \text { of pyridyl). }\end{array}$ \\
\hline $16 d$ & $\begin{array}{l}481(4), 454(28), 430(55), 378(4), 354(100), \\
347(86), 303(20), 267(46), 201(4), 137(14) \\
105(21), 77(4) .\end{array}$ & $2210(\mathrm{C} \equiv \mathrm{N})$ & $\begin{array}{l}3.02\left(3 \mathrm{H}, \mathrm{s}, \mathrm{CH}_{3}\right), 8.55(1 \mathrm{H}, \mathrm{s}, 4-\mathrm{H} \text { of pyridyl }) \\
8.32-8.30,7.79-7.65(15 \mathrm{H}, \mathrm{m}, \text { phenyl-H). }\end{array}$ \\
\hline $16 \mathrm{e}$ & $\begin{array}{l}\text { 476(5), 430(10), 368(55), 344(100), 327(4), } \\
268(8), 265(18), 201(2), 105(2) .\end{array}$ & $2206(\mathrm{C} \equiv \mathrm{N})$ & $\begin{array}{l}2.30\left(3 \mathrm{H}, \mathrm{s}, \mathrm{CH}_{3}\right), 3.38\left(3 \mathrm{H}, \mathrm{s}, \mathrm{CH}_{3}\right), 7.81(1 \mathrm{H}, \mathrm{s}, 5-\mathrm{H} \text { of } \\
\text { pyridyl }), 8.04(1 \mathrm{H}, \mathrm{dd}, J=1.53,1.51 \mathrm{~Hz}, 4-\mathrm{H} \text { of } \\
\text { thienyl-H), } 8.15(1 \mathrm{H}, \mathrm{d}, J=1.00, \mathrm{~Hz}, 3-\mathrm{H} \text { of thienyl-H), } \\
8.52(1 \mathrm{H}, \mathrm{d}, J=1.00, \mathrm{~Hz}, 5-\mathrm{H} \text { of thienyl-H), } 8.32-8.30 \text {, } \\
7.93-7.65(10 \mathrm{H}, \mathrm{m} \text {, phenyl-H). }\end{array}$ \\
\hline $16 f$ & $\begin{array}{l}\text { 498(5), 430(3), 368(7), 344(100), 268(5), } \\
\text { 201(2), 105(2), 77(2). }\end{array}$ & $\begin{array}{l}1688(\mathrm{C}=\mathrm{O}) \\
2207(\mathrm{C} \equiv \mathrm{N})\end{array}$ & $\begin{array}{l}2.32\left(3 \mathrm{H}, \mathrm{s}, \mathrm{CH}_{3}\right), 2.82\left(3 \mathrm{H}, \mathrm{s}, \mathrm{CH}_{3}\right), 8.84(1 \mathrm{H}, \mathrm{s}, 4-\mathrm{H} \text { of } \\
\text { pyridyl }), 8.41-8.36,8.30-8.17(15 \mathrm{H}, \mathrm{m}, \text { phenyl-H). }\end{array}$ \\
\hline
\end{tabular}

\footnotetext{
a Abbreviations: s: singlet; d: doublet; m: multiplet.
} 
of glacial acetic acid afforded the 6-[(1,5-diphenyl- $1 H$ pyrazol-4-yl)thio]-pyrimidine 19 (Scheme 4). The structure of pyrazole derivative 19 was established on the basis of their elemental analysis and spectral data. The IR spectra of compound 19 indicated the absence of the $\mathrm{C}=\mathrm{O}$ group and showed the characteristic absorption bands at $2201 \mathrm{~cm}^{-1}$ for the $\mathrm{C} \equiv \mathrm{N}$ group. The ${ }^{1} \mathrm{H}-\mathrm{NMR}$ spectra $\left(\mathrm{CF}_{3} \mathrm{COOD}\right)$ of compound 19 revealed a sharp singlet at $\delta 7.76(1 \mathrm{H}, \mathrm{s})$ assigned to the hydrogen attached at $\mathrm{C}_{3}$ of pyrazole ring and at $\delta 8.25-8.23,7.81-7.79,7.70-7.57(15 \mathrm{H}, \mathrm{m})$ assigned to the phenyl protons, which was also confirmed by the mass spectrum $m / z 445\left(\mathrm{M}^{+}\right)$. The formation of compound 19 would involve an initial nucleophilic substitution of the amino group in phenylhydrazine $\mathbf{1 8}$ to the activated double bond in enaminone derivative 17 , followed by deamination, to form the intermediate $\mathbf{1 9}^{\prime}$, which then undergoes intramolecule cyclization via loss of water [31] affording the final product 19. Next, the bis-pyrimidine derivative 6-[(4-phenyl-pyrimidin-5-yl)thio]-5-cyano4-methyl-2-phenyl-pyrimidine 21 was also obtained by the intramolecular cyclization of compound 17 with formamide/formic acid in the presence of excess ammonium acetate. The ${ }^{1} \mathrm{H}-\mathrm{NMR}$ spectra $\left(\mathrm{CF}_{3} \mathrm{COOD}\right)$ of compound $\mathbf{2 1}$ revealed additional two sharp singlets at $\delta 8.21(1 \mathrm{H}, \mathrm{s})$ and $8.26(1 \mathrm{H}, \mathrm{s})$ assigned to the hydrogen attached at $\mathrm{C}_{6}$ and $\mathrm{C}_{2}$ of pyrimidine ring and at $\delta 8.70-8.69,8.24-8.02(10 \mathrm{H}, \mathrm{m})$ assigned to the phenyl protons, which was also confirmed by the mass spectrum $m / z 381\left(\mathrm{M}^{+}\right)$.

Finally, intramolecular cyclization of the enaminone derivative 17 with 2-amino-bezimidazole 22, 5-amino$1 H$-tetrazole 24, 3-amino-4-phenylazo-pyrazole 26 and 3-amino-4-methyl-6-phenyl-pyrazolo[3,4-d]pyrimidine 28 under acid conditions afforded the corresponding benzimidazolo[1,2-a]pyrimidine $\quad 23, \quad$ 1,2,3,4-tetrazolo[1,5- $a$ ] pyrimidine 25, azopyrazolo[1,5-a]pyrimidine 27, and pyrimido-[4',5':3,4]pyrazolo[1,5-a]pyrimidine 29 , respectively (Scheme 4). The mechanisms of compounds $\mathbf{2 3}, \mathbf{2 5}$, 27, and 29 are similar to compound 19 . The structures of compounds 23, 25, 27, and 29 were established on the basis of their elemental analysis and spectral data. For instance, the ${ }^{1} \mathrm{H}$ NMR spectra of compounds $\mathbf{2 3}$ and $\mathbf{2 7}$ revealed a sharp singlet at $\delta 8.53(1 \mathrm{H}, \mathrm{s})$ and at $\delta 8.00(1 \mathrm{H}, \mathrm{s})$, which assigned to the hydrogen attached at $\mathrm{C}_{2}$ of benzimidazolopyrimidine and at $\mathrm{C}_{5}$ of pyrazolopyrimidine ring, respectively.

\section{Conclusion}

In conclusion, 6-methylthio-pyrimidine 2, 6-(benzoylmethyl) thio-pyrimidine 13 and 2-[(5-cyano-4-methyl2-phenylpyrimidin-6-yl)thio]-3-dimethylamino-1-phenylprop-2-en-1-one 17 have been shown to be a useful building block for the synthesis of some new $\mathrm{N}$-cycloalkanes, morpholine, piperazines, pyridines, pyrazole, pyrimidine, benzimidazolo[1,2-a]pyrimidine, 1,2,3,4-tetrazolo[1,5- $a$ ] pyrimidine, azopyrazolo[1,5-a]pyrimidine, and pyrimido $\left[4^{\prime}, 5^{\prime}: 3,4\right]$ pyrazolo[1,5-a]pyrimidine, respectively. The structure of all newly synthesized compounds was established from their spectral data, elemental analysis, and the X-ray crystal analysis.

\section{Acknowledgments}

The authors are grateful to the highly valued instrument cent of National Taiwan Normal University for measuring the data of spectroscopy. They also want to thank National Science Council of Taiwan (NSC 97-2113-M-253-001) for their financial support.

\section{References}

[1] K. M. Amin, M. M. Hanna, H. E. Abo-Youssef, F. Riham, and R. F. George, "Synthesis, analgesic and anti-inflammatory activities evaluation of some bi-, tri- and tetracyclic condensed pyrimidines," European Journal of Medicinal Chemistry, vol. 44, no. 11, pp. 4572-4584, 2009.

[2] D. C. Kim, Y. R. Lee, B. S. Yang et al., "Synthesis and biological evaluations of pyrazolo[3,4- $d]$ pyrimidines as cyclin-dependent kinase 2 inhibitors," European Journal of Medicinal Chemistry, vol. 38, no. 5, pp. 525-532, 2003.

[3] S. Pandey, S. N. Suryawanshi, Suman Gupta, and V. M. L. Srivastava, "Synthesis and antileishmanial profile of some novel terpenyl pyrimidines," European Journal of Medicinal Chemistry, vol. 39, no. 11, pp. 969-973, 2004.

[4] D. A. Abou El Ella, M. M. Ghorab, E. Noaman, H. I. Heiba, and A. I. Khalil, "Molecular modeling study and synthesis of novel pyrrolo[2,3-d]pyrimidines and pyrrolotriazolopyrimidines of expected antitumor and radioprotective activities," Bioorganic and Medicinal Chemistry, vol. 16, no. 5, pp. 2391-2402, 2008.

[5] Q. Xueqi, B. L. Bokai, W. Qi, L. Deshui, and L. Xian-Fu, "Facile synthesis of novel mutual derivatives of nucleosides and pyrimidines by regioselectively chemo-enzymatic protocol," Bioorganic and Medicinal Chemistry, vol. 16, no. 9, pp. 5181-5188, 2008.

[6] D. A. Ibrahim and A. M. El-Metwally, "Design, synthesis, and biological evaluation of novel pyrimidine derivatives as CDK2 inhibitors," European Journal of Medicinal Chemistry, vol. 45, no. 3, pp. 1158-1166, 2010.

[7] L. F. Kuyper, J. M. Garvey, D. P. Baccanari, J. N. Champness, D. K. Stammers, and C. R. Beddell, "Pyrrolo[2,3-d]pyrimidines and pyrido[2,3-d]pyrimidines as conformationally restricted analogues of the antibacterial agent trimethoprim," Bioorganic and Medicinal Chemistry, vol. 4, no. 4, pp. 593-602, 1996.

[8] S. N. Sriharsha, S. Satish, S. Shashikanth, and K. A. Raveesha, "Design, synthesis and antibacterial activity of novel 1,3thiazolidine pyrimidine nucleoside analogues," Bioorganic and Medicinal Chemistry, vol. 14, no. 22, pp. 7476-7481, 2006.

[9] A. Padmaja, T. Payani, G. D. Reddy, G. Dinneswara Reddy, and V. Padmavathi, "Synthesis, antimicrobial and antioxidant activities of substituted pyrazoles, isoxazoles, pyrimidine and thioxopyrimidine derivatives," European Journal of Medicinal Chemistry, vol. 44, no. 11, pp. 4557-4566, 2009.

[10] B. Ramesh and C. M. Bhalgat, "Novel dihydropyrimidines and its pyrazole derivatives: synthesis and pharmacological screening," European Journal of Medicinal Chemistry, vol. 46, no. 5, pp. 1882-1891, 2011. 
[11] E. A. Meade, M. Sznaidman, G. T. Pollard, L. M. Beauchamp, and J. L. Howard, "Anxiolytic activity of analogues of 4benzylamino-2-methyl-7H- pyrrolo[2,3-d]pyrimidines," European Journal of Medicinal Chemistry, vol. 33, no. 5, pp. 363-374, 1998.

[12] J. Y. Kim, D. Kim, S. Y. Kang et al., "Arylpiperazine-containing pyrimidine 4-carboxamide derivatives targeting serotonin 5HT2A, 5-HT2C, and the serotonin transporter as a potential antidepressant," Bioorganic and Medicinal Chemistry Letters, vol. 20, no. 22, pp. 6439-6442, 2010.

[13] E. P. D. S. Falcãoa, S. J. de Melo, R. M. Srivastava, M. T. J. D. A. Catanho, and S. C. Do Nascimento, "Synthesis and antiinflammatory activity of 4-amino-2-aryl-5-cyano-6-3- and 4-(N-phthalimidophenyl) pyrimidines," European Journal of Medicinal Chemistry, vol. 41, no. 2, pp. 276-282, 2006.

[14] K. S. Gudmundsson, B. A. Johns, and J. Weatherhead, "Pyrazolopyrimidines and pyrazolotriazines with potent activity against herpesviruses," Bioorganic and Medicinal Chemistry Letters, vol. 19, no. 19, pp. 5689-5692, 2009.

[15] M. P. Clark, K. M. George, R. G. Bookland et al. et al., "Development of new pyrrolopyrimidine-based inhibitors of Janus kinase 3 (JAK3)," Bioorganic and Medicinal Chemistry Letters, vol. 17, no. 5, pp. 1250-1253, 2007.

[16] N. Kumar, A. Chauhan, and S. Drabu, "Synthesis of cyanopyridine and pyrimidine analogues as new anti-inflammatory and antimicrobial agents," Biomedicine and Pharmacotherapy, vol. 65 , no. 5, pp. 375-380, 2011.

[17] J. M. Quintela, C. Peinador, L. González et al., "6Dimethylamino $1 \mathrm{H}$-pyrazolo[3,4- $d]$ pyrimidine derivatives as new inhibitors of inflammatory mediators in intact cells," Bioorganic and Medicinal Chemistry, vol. 11, no. 6, pp. 863-868, 2003.

[18] B. Tozkoparan, M. Ertan, P. Kelicen, and R. Demirdamar, "Synthesis and anti-inflammatory activities of some thiazolo[3,2a]pyrimidine derivatives," Farmaco, vol. 54, no. 9, pp. 588-593, 1999.

[19] J. P. Zhou, Y. W. Ding, H. B. Zhang, L. Xu, and Y. Dai, "Synthesis and anti-inflammatory activity of imidazo[1,2-a]pyrimidine derivatives," Chinese Chemical Letters, vol. 19, no. 6, pp. 669-672, 2008.

[20] H. S. Choi, Z. Wang, W. Richmond et al. et al., "Design and synthesis of $7 \mathrm{H}$-pyrrolo[2,3-d]pyrimidines as focal adhesion kinase inhibitors. Part 2," Bioorganic and Medicinal Chemistry Letters, vol. 16, no. 10, pp. 2689-2692, 2006.

[21] I. Devesa, M. J. Alcaraz, R. Riguera, and M. L. Ferrándiz, “A new pyrazolo pyrimidine derivative inhibitor of cyclooxygenase-2 with anti-angiogenic activity," European Journal of Pharmacology, vol. 488, no. 1-3, pp. 225-230, 2004.

[22] S. M. Sondhi, N. Singh, M. Johar, and A. Kumar, "Synthesis, anti-inflammatory and analgesic activities evaluation of some mono, bi and tricyclic pyrimidine derivatives," Bioorganic and Medicinal Chemistry, vol. 13, no. 22, pp. 6158-6166, 2005.

[23] Y. W. Ho and W. H. Yao, "Reactions of 6-acetyl-4-methyl5-(1-pyrrolyl)-2-phenylthieno-[2,3-d] pyrimidine in heterocyclic synthesis: Convenient route to some Schiff's bases, chalcones, pyridines, pyridin-2 $(1 \mathrm{H})$-ones and $2 \mathrm{H}$-pyran-2-one derivatives incorporating a 5-(1-pyrrolyl)-2-phenylthieno[2,3d]pyrimidine moiety," Journal of the Chinese Chemical Society, vol. 52, no. 2, pp. 313-325, 2005.

[24] Y. W. Ho, "5-(1-Pyrrolyl)-2-phenylthieno[2,3-d]pyrimidine as building block in heterocyclic synthesis: Novel synthesis of some pyrazoles, pyrimidines, imidazo[1,2-a]pyrimidines, pyrazolo[1,5-a]pyrimidines, pyrido-(pyrimido) pyrazolo[1,5a]pyrimidines, 1,2,4-triazolo[1,5-a]pyrimidine and a 1,2,3,4tetrazolo[1,5-a]pyrimidine derivative," Journal of the Chinese Chemical Society, vol. 54, no. 4, pp. 1075-1085, 2007.

[25] Y. W. Ho and M. C. Suen, "Synthesis and structure of novel thieno[2,3-d]pyrimidine derivatives containing 1,3,4oxadiazole moiety," Journal of the Chinese Chemical Society, vol. 56, no. 2, pp. 408-415, 2009.

[26] Y. W. Ho and C. T. Yao, "Synthesis of some new 6,8disubstituted 7,8-dihydropyrimido[2,3:4,3] pyrazolo[1,5- $a]$ pyrimidines and 6,7,8-trisubstituted pyrimido [2,3:4,3]pyrazolo[1, 5-a]pyrimidine derivatives," Journal of the Chinese Chemical Society, vol. 50, no. 2, pp. 283-296, 2003.

[27] F. Karci and A. Demirçali, "Synthesis of disazo pyrazolo[1,5a]pyrimidines," Dyes and Pigments, vol. 74, no. 2, pp. 288-297, 2007.

[28] XSCANS, Release, 2.1, Siemens Energy \& Automation, Inc, Madison, Wis, USA, 1995.

[29] SMART/SAINT/ASTRO, Release, 4.03, Siemens Energy \& Automation, Inc, Madison, Wis, USA, 1995.

[30] L. Roman, V. Olena, H. Serhiy, Z. Lucjusz, and G. Andrej, "New 5-substituted thiazolo[3,2-b][1,2,4]triazol-6-ones: synthesis and anticancer evaluation," European Journal of Medicinal Chemistry, vol. 42, no. 5, pp. 641-648, 2007.

[31] K. M. Dawood, A. M. Farag, and E. A. Ragab, "A facile access to polysubstituted bipyrazoles and pyrazolylpyrimidines," Journal of the Chinese Chemical Society, vol. 51, no. 4, pp. 853-857, 2004. 

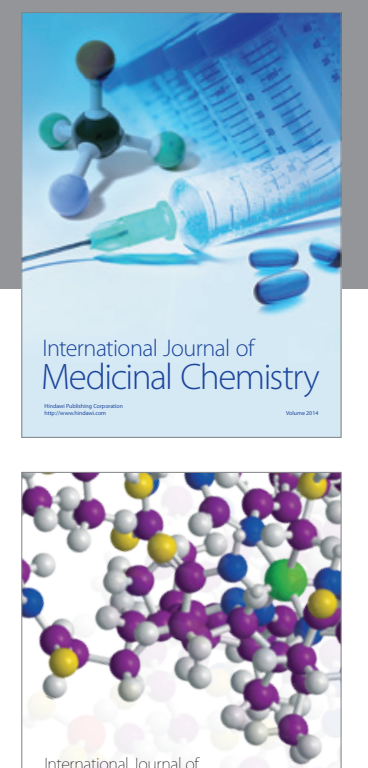

\section{Carbohydrate} Chemistry

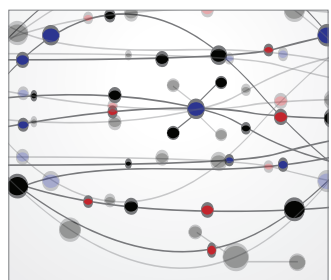

The Scientific World Journal
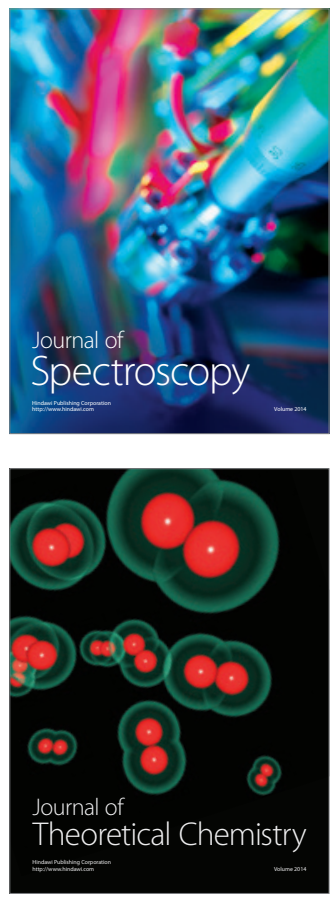
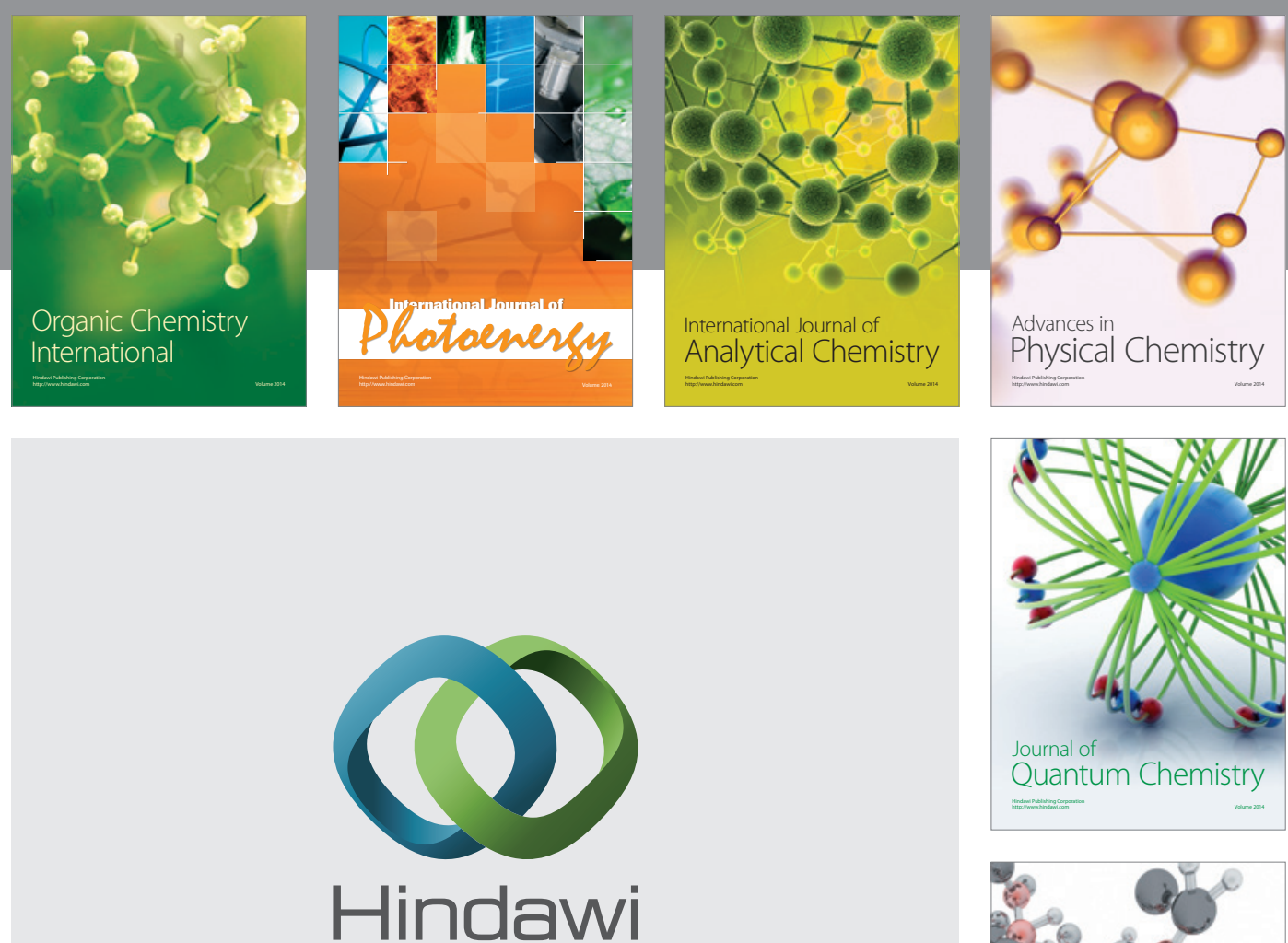

Submit your manuscripts at

http://www.hindawi.com

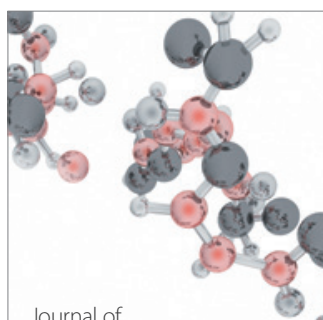

Analytical Methods

in Chemistry

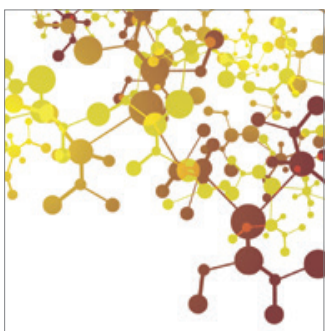

Journal of

Applied Chemistry

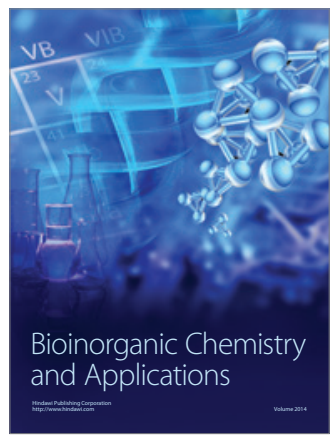

Inorganic Chemistry
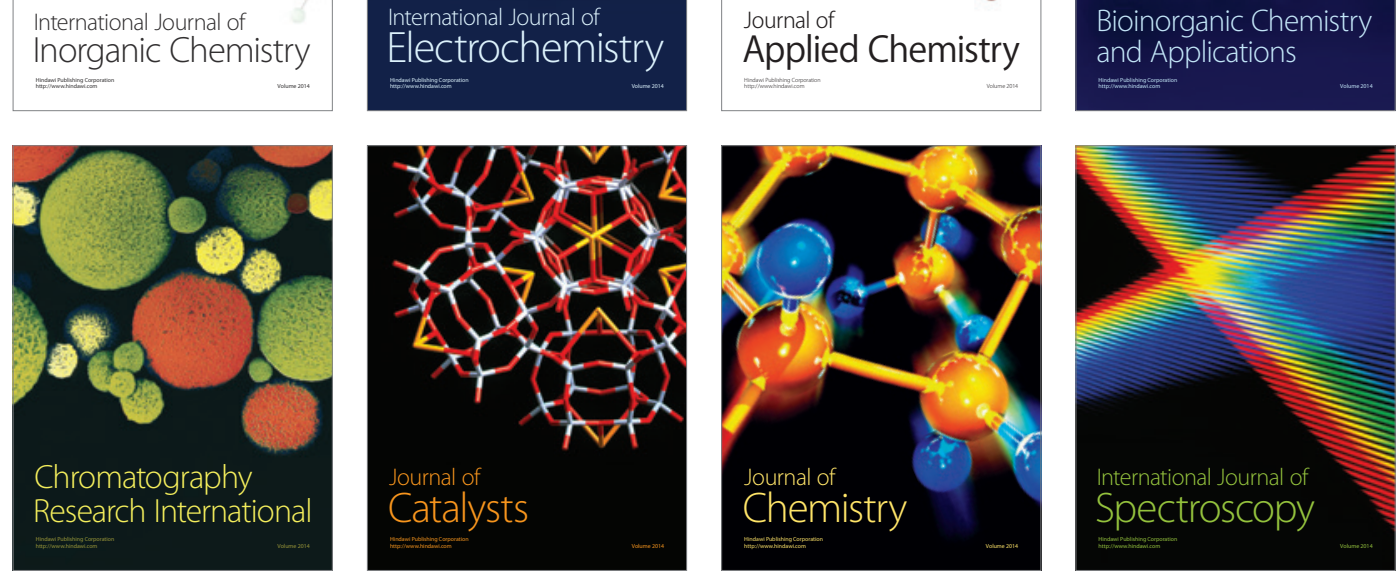